\title{
Cascade Delayed Controller Design for a Class of Underactuated Systems
}

\author{
G. Ochoa-Ortega $\mathbb{D}^{1},{ }^{1}$ R. Villafuerte-Segura $\mathbb{D}^{2},{ }^{2}$ A. Luviano-Juárez, ${ }^{3}$ M. Ramírez-Neria, ${ }^{4}$ \\ and N. Lozada-Castillo ${ }^{3}{ }^{3}$ \\ ${ }^{1}$ Universidad Politéenica del Valle de México, Av. Mexiquense s/n Colonia Villa Esmeralda, Tultitlán 54910, Mexico \\ ${ }^{2}$ Universidad Autónoma del Estado de Hidalgo, CITIS, Carretera Pachuca-Tulancingo km. 4.5, Col. Carboneras, \\ Mineral de la Reforma 42184, Mexico \\ ${ }^{3}$ Instituto Politécnico Nacional-UPIITA, Av. IPN 2580 Colonia Barrio la Laguna Ticomán, Ciudad de México 07340, Mexico \\ ${ }^{4}$ InIAT Universidad Iberoamericana Ciudad de México, Prolongación Paseo de la Reforma 880, Colonia Lomas de Santa Fé, \\ Ciudad de México CP 01219, Mexico
}

Correspondence should be addressed to R. Villafuerte-Segura; villafuerte@uaeh.edu.mx

Received 16 April 2020; Revised 11 July 2020; Accepted 22 July 2020; Published 25 August 2020

Academic Editor: Alejandro F. Villaverde

Copyright ( $) 2020$ G. Ochoa-Ortega et al. This is an open access article distributed under the Creative Commons Attribution License, which permits unrestricted use, distribution, and reproduction in any medium, provided the original work is properly cited.

\begin{abstract}
In this paper, a delayed control strategy for a class of nonlinear underactuated fourth-order systems is developed. The proposal is based on the implementation of the tangent linearization technique, differential flatness, and a study of the $\sigma$-stabilization of the characteristic equation of the closed-loop system. The tangent linearization technique allows obtaining a local controllability property for the analyzed class of systems. Also, it can reduce the complexity of the global control design, through the use of a cascade connection of two second-order controllers instead of designing a global controller of the fourth-order system. The stabilizing behavior of the delayed controller design is supported by the $\sigma$-stability criterion, which provides the controller parameter selection to reach the maximum exponential decay rate on the system response. To illustrate the efficiency of the theoretical results, the proposal is experimentally assessed in two cases of study: a flexible joint system and a pendubot.
\end{abstract}

\section{Introduction}

The control of underactuated systems has attracted some attention from the academic community, as noticed in the growing list of articles and new approaches to solve the problems of estimation, regulation, and trajectory tracking involving both linear and nonlinear underactuated systems. In the literature, there are a variety of control strategies, including controlled Lyapunov functions $[1,2]$, energybased control [3-5], passivity approaches [6, 7], active disturbance rejection [8-10], planning algorithms, and feedback stabilization schemes [11].

One of the most important problems of nonlinear underactuated systems is the fact that the controllability property may be subject to singularities (ill-conditioned relative degree [12]), which conditions the controllable (normal) forms of the models to a certain class of systems [13]. An alternative to locally overcome the aforementioned problem is the use of the tangent linearization technique, since the tangent linearized models of an important class of underactuated systems are differentially flat [9]. The last fact means that the linearized systems are controllable and there exists a set of variables, named flat outputs, which can characterize them [14]. Besides the flatness of the linearized system, another important advantage for this class of systems is the called cascade form [15], which allows finding a relation between $2 n$-th-order time derivatives (fourthorder, sixth-order, and so on) of the flat output and a measurable variable of the system. This form can reduce the complexity of the global control design in which, instead of designing a global controller of a $2 n$-th-order system, the task can be the cascade connection of second-order 
controllers, which is especially important for sensitive systems or noise amplification effects due to high-order observer designs, among others. In particular, for the case of fourth-order systems, this scheme allows, instead of stabilizing a fourth-order integrator, implementing a cascade control arrangement of two second-order systems, for instance, classic controllers with derivative action. The implementation of the derivative action has at least three natural disadvantages. Firstly, the estimation algorithms typically increase the controller design. Secondly, the application of filters or compensators often increases the order of the closed-loop system. Thirdly, the use of measurement tools (encoders) is usually very noisy. An alternative is the use of delayed controllers, which means to deliberately include retarded actions (time delays) in the controller. Delayed controllers have better performance in practical applications compared to controllers with derivative actions [16]. Since these controllers type noise attenuation, they do not require estimators or filters to approximate the time derivative, providing soft control signals which do not damage actuators, and their numerical implementation is computationally more efficient than other low-order controllers. Moreover, a delayed controller has a simple structure which is easy to implement, like classic controllers.

The deliberate use of retarded actions to stabilize a system is a topic that has been investigated in recent decades, among which are the pioneering contributions of [17-28]. Concerning derivative-free control based schemes, such as the proportional retarded (PR) scheme [19, 25, 29-31], which consists in using the relation between the time derivative approximations in terms of a differential difference equation, involving a time delay. This approximation is taken as a baseline to avoid using a derivative compensation, but a proportional retarded one.

The use of delayed controllers has been previously studied for the stabilization of chains of integrators. It has been proven [32-34] that a chain of $n$ integrators cannot be stabilized with less than $n$ delay blocks, which increases significantly the complexity of the closed-loop system. The migration of a double imaginary characteristic root to the left half-plane or the right half-plane under the variation of two parameters of a quasi-polynomial is given in [35]. However, in none of the above proposals, there are criteria or methodologies to explicitly tune the controller gains.

For the class of underactuated systems satisfying the cascade property, the use of a cascade control structure of PR controllers (delayed controller) can be a derivative-free alternative of stabilizing control, including the complete dynamics (some reported schemes based on the linearization deal with a part of the dynamics such as the switched control [36]). However, the existing analyses are restricted to a class of second-order LTI systems, and the system to analyze becomes a cascade of two-order systems, whose analysis cannot be considering the isolated dynamics but the cascade structure.

The stability analysis of delayed controllers can be commonly addressed on the study of the relative stability of degree $\sigma$ of the characteristic function, also known as $\sigma$-stability. This approach in conjunction with the
D-decomposition methodology (see [37-40]) can lead us to obtain conditions, under which the system response can reach the maximal exponential decay rate.

Thus, the contributions of this manuscript are listed as follows:

(1) It analyzed a class of underactuated systems, especially those whose tangent linearization is controllable (flat). Moreover, it developed a controller through the use of the cascade property in the flat linearized system, which allows reducing the complexity of the control loops.

(2) The control approach consists in using a cascade structure of proportional retarded controllers in each of the second-order structures derived from the cascade system. In this proposal, the fourth-order system is controlled by a tandem array of PR controllers in which two delays are used for the main structure.

(3) The stability analysis considers the complete fourthorder structure and provides the tuning conditions for the controller design and, moreover, provides competitive experimental results, which denote the importance of using a more complex controller with the aim of obtaining appropriate results for noisy measurements, in which additional filtering schemes are demanded. In addition, the tuning procedure is reported and the involved numerical algorithms are provided for practitioners.

(4) The combination of the aforementioned concepts provides an alternative control approach for a class of fourth-order underactuated systems subject to noisy measurements, with a possible extension to the highest-order systems.

(5) The analysis and synthesis of a cascade proportional retarded controllers for a class of underactuated systems in cascade structure are proposed (Figure 1). Also, analytic conditions, on the parameters of the controller, to reach the maximal exponential decay rate of the system response are proposed.

(6) Experimental implementations of the proposal, which validate its practical effectiveness, are also proposed. Two classic challenging systems were used for the assessment: a rotatory flexible link and a pendubot.

The remaining of the contribution is organized as follows. In Section 2, the basic concepts related to time-delay systems and differentially flatness are presented. In Section 3 , a methodology based on the differential flatness to represent a class of fourth-order underactuated systems as a cascade arrangement of two-order systems is presented. The main contributions of the present manuscript are introduced in Section 4, where the $\sigma$-stability of the system is analyzed and analytic conditions to reach the maximal exponential decay rate of the system response are provided. Section 5 is devoted to the implementation results in trajectory tracking tasks. The article ends with some concluding remarks. 


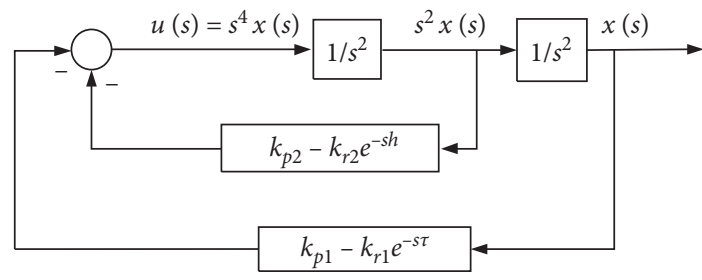

FIGURE 1: Schematic of the PR controller for a cascade structure.

Notation. Given a vector $x(t) \in \mathbb{R}^{n}, x^{\top}(t)$ denotes its transpose and $\|x(t)\|$ stands for the Euclidean norm. Let $s \in \mathbb{C}$; then, $\operatorname{Re}(s)$ and $\operatorname{Im}(s)$ denote its real and imaginary parts, respectively. For a function $x(t), x^{(i)}(t), i \in \mathbb{Z}^{+}$denotes the $i$-th time derivative of the function.

\section{Mathematical Preliminaries}

In this section, we introduce some basic concepts, concerning both time-delay systems and differentially flat systems, necessary for the development of this contribution.

2.1. Differentially Flat Systems. In this subsection, the flatness concept for single-input single-output (SISO) systems of nonlinear and linear types is taken. Further comprehensive information can be taken from [14, 41, 42] and references therein.

Definition 1. Let us consider a nonlinear system of the following form:

$$
\dot{x}(t)=f(x(t), u(t)),
$$

where $x(t) \in \mathbb{R}^{n}, \quad u(t) \in \mathbb{R}$, and $f(\cdot)=\left[f_{1}(\cdot) f_{2}(\cdot)\right.$ $\left.\cdots f_{n}(\cdot)\right]^{\top}$ is a smooth function of $x(t), u(t)$, and the rank of the Jacobian matrix, and $(\partial f(\cdot)) /(\partial u(t))$ is equal to 1 . System (1) is said to be differentially flat if there exists a differential function, denoted as $y(t) \in \mathbb{R}$, given by

$$
y(t)=r\left(x(t), u(t), \dot{u}(t), \ddot{u}(t), \ldots, u^{(\alpha)}(t)\right), \quad \alpha \in \mathbb{Z}^{+},
$$

which completely differentially parametrizes all the variables of the system. This means that states, inputs, and original output variables in the system can be written as differential functions of the flat output $y(t)$, that is,

$$
\begin{aligned}
& x(t)=\phi\left(y(t), \dot{y}(t), \ldots, y^{(\gamma)}(t)\right), \\
& u(t)=\psi\left(y(t), \dot{y}(t), \ldots, y^{(\gamma+1)}(t)\right), \quad \gamma \in \mathbb{Z}^{+} .
\end{aligned}
$$

Flatness is a structural property of the system that trivializes the exact linearization procedure of a nonlinear system even when it is not expressed in an affine form. It also allows an easier manner of designing control laws in trajectory tracking tasks, by taking advantage of the differential parametrization. For the case of linear SISO systems, the flatness property can be directly related to the controllability of the system.
Definition 2. Consider the linear time-invariant SISO system:

$$
\dot{x}(t)=A x(t)+b u(t)
$$

with $A \in \mathbb{R}^{n \times n}, b \in \mathbb{R}^{n}, x(t) \in \mathbb{R}^{n}$, and $u(t) \in \mathbb{R}$. Let the pair $(A, b)$ be controllable, that is, the controllability matrix:

$$
K_{c}=\left[\begin{array}{lllll}
b & A b & A^{2} b & \cdots & A^{n-1} b
\end{array}\right],
$$

which has full rank $n$. Then, it is said that system (4) is controllable; hence, it is differentially flat with the flat output given by

$$
y(t)=\left[\begin{array}{llll}
0 & 0 & \cdots & 1
\end{array}\right] K_{c}^{-1} x(t) .
$$

2.2. Time-Delay Systems. In the present section, some definitions and general results concerning linear time-delay systems are introduced; for these purposes, it is considered a basic system of the following form:

$$
\dot{x}(t)=A_{0} x(t)+A_{1} x(t-\tau)+A_{2} x(t-h), \quad t \geq 0 .
$$

Here, $A_{0}, A_{1} \in \mathbb{R}^{n \times n}$, and $\tau, h \in \mathbb{R}^{+}$are time delays. Now, some stability concepts related to system (7) are presented.

Definition 3 (see [43]). The solution $x(t, \varphi)$ of a system of the form (7) is said to be exponentially stable, if there exist $L, \sigma \in \mathbb{R}^{+}$such that the following inequality holds:

$$
\|x(t, \varphi)\| \leq L \mathrm{e}^{-\sigma t}\|\varphi\|_{H}, \quad t \geq 0 .
$$

Here, $H=\max \{\tau, h\}, \varphi:[-H, 0] \longrightarrow \mathfrak{C}$ denotes the initial function and $\mathfrak{C}:=C\left([-H, 0], \mathbb{R}^{n}\right)$ is a Banach space of continuous functions in $[-H, 0]$ with the norm $\|\varphi\|_{H}=\max _{\theta \in[-H, 0]}\|\varphi(\theta)\|$.

Definition 4 (see [43]). A $s_{0} \in \mathbb{C}$ is said to be an eigenvalue of system (7) if it is a root of the characteristic function; that is,

$$
\mathrm{Q}\left(s_{0}, \tau, h\right)=\operatorname{det}\left(s_{0} I-A_{0}-A_{1} \mathrm{e}^{-s_{0} \tau}-A_{2} \mathrm{e}^{-s_{0} h}\right)=0 .
$$

The set of all the distinct eigenvalues of the system is called the spectrum of system (7) and this is denoted as $\Lambda(Q)$.

An equivalent definition of exponential stability is given hereinafter.

Definition 5 (see [43]). System (7) is exponentially stable if and only if the spectrum of the system lies in the open left half-plane of the complex plane; that is, $\operatorname{Re}\left(s_{j}\right)<0$, $\forall s_{j} \in \Lambda(Q)$.

Definition 6. A linear time-delay system of the form (7) is said to be $\sigma$-stable, if

$$
s^{*}=-\sigma,
$$

for $\sigma \in \mathbb{R}^{+}$and $s^{*}=\max _{j=1, \ldots, \infty}\left\{\operatorname{Re}\left(s_{j}\right): s_{j} \in \Lambda(Q)\right\}$.

From these definitions, note that $\sigma$-stability ensures exponential stability with the decay rate $\sigma$ in the system response (7). 
In the following section, a class of fourth-order underactuated systems is described.

\section{Preliminary Results}

Euler-Lagrange underactuated systems of the fourth order can generally be described by

$$
P(q(t)) u(t)=M(q(t)) \ddot{q}(t)+C(q(t), \dot{q}(t)) \dot{q}(t)+g(q(t)),
$$

where $q(t):=\left[q_{1}(t) q_{2}(t)\right]^{\top}$ represents the generalized coordinates, $M(q(t)) \in \mathbb{R}^{2 \times 2}$ is the inertia matrix, and $C(q(t), \dot{q}(t)) \in \mathbb{R}^{2 \times 2}$ describes the Coriolis matrix and centrifugal forces. Let $g(q(t)) \in \mathbb{R}^{2}$ be the vector of gravitational forces and $P(q(t)) \in \mathbb{R}^{2}$ is the vector mapping the external forces. Finally, $u(t) \in \mathbb{R}$ denotes the control input.

Systems of the form (11) can be rewritten in a state-space representation as follows:

$$
\begin{aligned}
& \dot{x}_{a}(t)=x_{b}(t), \\
& \dot{x}_{b}(t)=f\left(x_{a}(t), t x_{b} n(t)\right)+\zeta\left(x_{a}(t)\right) u(t),
\end{aligned}
$$

where $x_{a}(t)$ stands for the vector of joint positions $\left[\begin{array}{ll}q_{1}(t) & q_{2}(t)\end{array}\right]^{\top}, x_{b}(t)$ denotes the articular velocities vector $\left[\begin{array}{ll}\dot{q}_{1}(t) & \dot{q}_{2}(t)\end{array}\right]^{\top}$, and

$$
\begin{aligned}
f\left(x_{a}(t), x_{b}(t)\right) & =-M(q(t))^{-1}[C(q(t), \dot{q}(t)) \dot{q}(t)+g(q(t))], \\
\zeta\left(x_{a}(t)\right) & =M(q(t))^{-1} P(q(t)) .
\end{aligned}
$$

After a slight rearrangement of the state vector as $x(t)=$

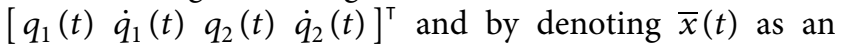
equilibrium point of the nonlinear representation (12), a tangent linearization around the equilibrium point $\bar{x}(t)$ is given by

$$
\dot{x}_{\delta}(t)=A x_{\delta}(t)+B u_{\delta}(t) .
$$

Here, $x_{\delta}(t)=x(t)-\bar{x}(t)$ and $u_{\delta}(t)=u(t)-\bar{u}(t)$. The controllability matrix of system (14) is given by

$$
K_{c}=\left[\begin{array}{llll}
b A & b A^{2} & b A^{3} & b
\end{array}\right] .
$$

Under the assumption that $\operatorname{det}\left(K_{c}\right) \neq 0$, the system is controllable and hence, according to $[14,42]$, differentially flat. The flat output can be obtained as follows:

$$
y_{f}(t)=C_{f} x_{\delta}(t)
$$

where $C_{f} \in \mathbb{R}^{n}$ and defined as

$$
C_{f} \epsilon:=\epsilon\left[\begin{array}{llll}
0 & 0 & 0 & 1
\end{array}\right] K_{c}^{-1} \text {. }
$$

Here, $\epsilon \in \mathbb{R} /\{0\}$ is an arbitrary constant. In order to determine the relative degree of system (14), we first need to compute the high-order time derivatives. To this end, notice that the following relations hold:

$$
C_{f} b=C_{f} A b=C_{f} A^{2} b=0, \quad C_{f} A^{3} b \neq 0,
$$

and the time derivatives of the flat output are given by

$$
\begin{aligned}
& y_{f}(t)=C_{f} x_{\delta}(t), \\
& \dot{y}_{f}(t)=C_{f} A x_{\delta}(t), \\
& \ddot{y}_{f}(t)=C_{f} A^{2} x_{\delta}(t), \\
& y_{f}^{(3)}(t)=C_{f} A^{3} x_{\delta}(t), \\
& y_{f}^{(4)}(t)=C_{f} A^{4} x_{\delta}(t)+C_{f} A^{3} b u_{\delta}(t) .
\end{aligned}
$$

Since $C_{f} A^{3} b \neq 0$, then, the relative degree of the system is four. It is worth noting that the even time derivatives of $y_{f}(t)$ can be expressed as a linear combination of the flat output, which correspond to those variables that can be measured and coincide with the vector of generalized positions $q(t)$.

Now, let us define the output estimation error as

$$
e_{i}(t):=y_{f}^{(i-1)}(t)-y_{f}^{*(i-1)}(t), \quad i=1, \ldots, 4 .
$$

Here, $y_{f}^{*}(t)$ denotes the desired output. Then, the error dynamics is governed by

$$
\begin{aligned}
& \dot{e}_{1}(t)=e_{2}(t), \\
& \dot{e}_{2}(t)=e_{3}(t), \\
& \dot{e}_{3}(t)=e_{4}(t), \\
& \dot{e}_{4}(t)=y_{f}^{(4)}(t)-y_{f}^{*(4)}(t),
\end{aligned}
$$

by proposing the auxiliary control

$$
u_{\delta}(t)=\left(C_{f} A^{3} b\right)^{-1}\left[u(t)-C_{f} A^{4} x_{\delta}(t)+y_{f}^{*(4)}(t)\right],
$$

and then (21) can be expressed as a fourth-order integration chain; that is,

$$
\begin{aligned}
& \dot{e}_{1}(t)=e_{2}(t), \\
& \dot{e}_{2}(t)=e_{3}(t), \\
& \dot{e}_{3}(t)=e_{4}(t), \\
& \dot{e}_{4}(t)=u(t) .
\end{aligned}
$$

Now, a delayed controller $u(t)$ that fulfills the cascade control structure proposed in Figure 1 is given as follows:

$$
u(t)=-k_{p_{1}} e_{1}(t)+k_{r_{1}} e_{1}(t-\tau)-k_{p_{2}} e_{3}(t)+k_{r_{2}} e_{3}(t-h),
$$

where $\tau, h \in \mathbb{R}^{+}$are the delays and $k_{p_{1,2}}, k_{r_{1,2}} \in \mathbb{R}^{+}$are the control gains. Thus, the closed-loop representation of system (23) is now

$$
\dot{e}(t)=A_{0} e(t)+A_{1} e(t-\tau)+A_{2} e(t-h) .
$$


Here,

$$
\begin{aligned}
& A_{0}=\left[\begin{array}{rrrr}
0 & 1 & 0 & 0 \\
0 & 0 & 1 & 0 \\
0 & 0 & 0 & 1 \\
-k_{p_{1}} & 0 & -k_{p_{2}} & 0
\end{array}\right], \\
& A_{1}=\left[\begin{array}{rrrr}
0 & 0 & 0 & 0 \\
0 & 0 & 0 & 0 \\
0 & 0 & 0 & 0 \\
k_{r_{1}} & 0 & 0 & 0
\end{array}\right], \\
& A_{2}=\left[\begin{array}{llll}
0 & 0 & 0 & 0 \\
0 & 0 & 0 & 0 \\
0 & 0 & 0 & 0 \\
0 & 0 & k_{r_{2}} & 0
\end{array}\right],
\end{aligned}
$$

and $e(t)=\left[\begin{array}{llll}e_{1}(t) & e_{2}(t) & e_{3}(t) & e_{4}(t)\end{array}\right]^{\top}$. Thus, the characteristic function or characteristic quasi-polynomial of system (25) is given by

$$
Q\left(s, k_{p_{1,2}}, k_{r_{1,2}}, \tau, h\right)=s^{4}+k_{p_{2}} s^{2}+k_{p_{1}}-k_{r_{1}} e^{-s \tau}-s^{2} k_{r_{2}} e^{-s h} .
$$

\section{Main Results}

In the present section, analytic conditions on the parameters of the delayed controller (24) are presented. Here, a general form of quasi-polynomial (27) is considered:

$$
Q\left(s, k_{p_{1,2}}, k_{r_{1,2}}, \tau, h\right)=P_{1}\left(s, k_{p_{1,2}}\right)+P_{2}\left(s, k_{r_{1}}\right) e^{-s \tau}+P_{3}(s) k_{r_{2}} e^{-s h},
$$

where $P_{i}(\cdot), i=1,2,3$, are polynomials with real coefficients which satisfy the following degree condition: $\operatorname{deg}\left(P_{1}(\cdot)\right)>\operatorname{deg}\left(P_{2,3}(\cdot)\right)$. In order to analyze the $\sigma$-stability of the quasi-polynomial (28), the change of variable $s \longrightarrow(s-\sigma)$ is considered; thus, $(28)$ is now in the following form:

$$
\begin{aligned}
Q\left(s-\sigma, k_{p_{1,2}}, k_{r_{1,2}}, \tau, h\right):= & Q_{\sigma}\left(s, k_{p_{1,2}}, k_{r_{1,2}}, \tau, h\right) \\
= & P_{\sigma_{1}}\left(s, k_{p_{1,2}}\right)+P_{\sigma_{2}}\left(s, k_{r_{1}}\right) e^{-\tau(s-\sigma)} \\
& +P_{\sigma_{3}}(s) e^{\sigma h} k_{r_{2}} e^{-s h} .
\end{aligned}
$$

Based on the $\mathscr{D}$-decomposition methodology [44], the $\sigma$-stability regions of the modified characteristic function (29) can be now obtained. This procedure will play a key role in determining the analytic conditions to obtain the maximal decay rate $\sigma^{*}$.

Proposition 1. Let us consider a quasi-polynomial of the form (28), with given control parameters $k_{p_{1}}, k_{p_{2}}, k_{r_{1}}$, and $\tau$. Let $\sigma \in \mathbb{R}^{+}$be fixed; then, by defining

$$
\Gamma(s, \sigma)=P_{\sigma_{1}}\left(s, k_{p_{1,2}}\right)+P_{\sigma_{2}}\left(s, k_{r_{1}}\right) e^{-(s-\sigma) \tau},
$$

the $\sigma$-stability regions on the $\left(h, k_{r}\right)$ parametric space are bounded by the following conditions.

When $s=0$,

$$
k_{r_{2}}(h)=-\frac{\Gamma(0, \sigma)}{P_{\sigma_{3}}(0) e^{\sigma h}}, \quad h \in \mathbb{R}^{+} .
$$

When $s=i \omega, \omega \in \mathbb{R}^{+}$,

$$
\begin{array}{r}
h(\omega)=\frac{1}{\omega} \cot ^{-1}\left(-\frac{\operatorname{Re}\left((\Gamma(i \omega, \sigma)) /\left(P_{\sigma_{3}}(i \omega)\right)\right)}{\operatorname{Im}\left((\Gamma(i \omega, \sigma)) /\left(P_{\sigma_{3}}(i \omega)\right)\right)}\right)+\frac{\pi n}{\omega}, \\
n=0, \pm 1, \pm 2, \ldots,
\end{array}
$$

$$
k_{r_{2}}(h, \omega)=\frac{1}{e^{\sigma h} \sin (\omega h)} \operatorname{Im}\left(\frac{\Gamma(i \omega, \sigma)}{P_{\sigma_{3}}(i \omega)}\right) .
$$

Proof. It should be noted that the $\sigma$ crossing boundaries of the quasi-polynomial (28), in the parametric space $\left(h, k_{r}\right)$, are given by the critical crossings roots of quasi-polynomial (29), which occur when $s=0$ and $s= \pm i \omega$. In this regard, on the one hand, the solutions of quasi-polynomial (29) when $s=0$ are given by

$$
\begin{aligned}
0=\left.Q_{\sigma}\left(s, k_{p_{1,2}}, k_{r_{1,2}}, \tau, h\right)\right|_{s=0}= & P_{\sigma_{1}}\left(0, k_{p_{1,2}}\right)+P_{\sigma_{2}}\left(0, k_{r_{1}}\right) e^{\tau \sigma} \\
& +P_{\sigma_{3}}(0) e^{\sigma h} k_{r_{2}},
\end{aligned}
$$

or equivalently

$$
k_{r_{2}}=-\frac{P_{\sigma_{1}}\left(0, k_{p_{1,2}}\right)+P_{\sigma_{2}}\left(0, k_{r_{1}}\right) \mathrm{e}^{\tau \sigma}}{P_{\sigma_{3}}(0) \mathrm{e}^{\sigma h}} .
$$

On the other hand, the solutions of the quasi-polynomial (29) when $s=i \omega$ are given by

$$
\begin{aligned}
0= & \left.Q_{\sigma}\left(s, k_{p_{1,2}}, k_{r_{1,2}}, \tau, h\right)\right|_{s=i \omega} \\
= & P_{\sigma_{1}}\left(i \omega, k_{p_{1,2}}\right)+P_{\sigma_{2}}\left(i \omega, k_{r_{1}}\right) e^{-(i \omega-\sigma) \tau} \\
& +P_{\sigma_{3}}(s) e^{\sigma h} k_{r_{2}} e^{-i \omega h} .
\end{aligned}
$$

Thereby,

$$
-\frac{1}{k_{r_{2}} e^{\sigma h}}\left(\frac{\Gamma(i \omega, \sigma)}{P_{\sigma_{3}}(i \omega)}\right)=e^{-i \omega h}=\cos (\omega h)-i \sin (\omega h),
$$

from which

$$
\begin{aligned}
& \cos (\omega h)=-\frac{1}{k_{r_{2}} e^{\sigma h}} \operatorname{Re}\left(\frac{\Gamma(i \omega, \sigma)}{P_{\sigma_{3}}(i \omega)}\right), \\
& \sin (\omega h)=\frac{1}{k_{r_{2}} e^{\sigma h}} \operatorname{Im}\left(\frac{\Gamma(i \omega, \sigma)}{P_{\sigma_{3}}(i \omega)}\right) .
\end{aligned}
$$


From the above equation, it follows that

$$
\frac{\cos (\omega h)}{\sin (\omega h)} \operatorname{Im}\left(\frac{\Gamma(i \omega, \sigma)}{P_{\sigma_{3}}(i \omega)}\right)=-\operatorname{Re}\left(\frac{\Gamma(i \omega, \sigma)}{P_{\sigma_{3}}(i \omega)}\right) .
$$

Then, the solutions of (39) (with respect to h) are of the following form:

$$
\begin{array}{r}
h=\frac{1}{\omega} \cot ^{-1}\left(-\frac{\operatorname{Re}\left((\Gamma(i \omega, \sigma)) /\left(P_{\sigma_{3}}(i \omega)\right)\right)}{\operatorname{Im}\left((\Gamma(i \omega, \sigma)) /\left(P_{\sigma_{3}}(i \omega)\right)\right)}\right)+\frac{\pi n}{\omega}, \\
n=0, \pm 1, \pm 2, \ldots, \text { and } \omega \in \mathbb{R}^{+} .
\end{array}
$$

Finally, solving $k_{r_{2}}$ from (38), equation (33) follows.

In the following corollary, the $\sigma$-stability boundaries in the $\left(h, k_{r_{2}}\right)$ parametric space are written particularly for the quasi-polynomial (23), where $P_{1}\left(s, k_{p_{1,2}}\right)=s^{4}+k_{p_{2}} s^{2}+k_{p_{1}}$, $P_{2}\left(s, k_{r_{1}}\right)=-k_{r_{1}}$, and $P_{3}(s)=-s^{2}$.

Corollary 1. Consider the quasipolynomial of the form (23). Then, the $\sigma$-stability boundaries (31)-(33) look as follows.

For $s=0$,

$$
k_{r_{2}}(h)=\frac{k_{p_{2}} \sigma^{4}+k_{p_{2}} \sigma^{2}+k_{p_{1}}-k_{r_{1}} e^{\sigma \tau}}{\sigma^{2} e^{\sigma h}} .
$$

For $s=i \omega, \omega \in \mathbb{R}^{+}$,

$$
\begin{gathered}
h(\omega)=\frac{1}{\omega} \cot ^{-1}\left(-\frac{\Phi}{\Psi}\right)+\frac{\pi n}{\omega}, \\
k_{r_{2}}(h, \omega)=\frac{\Psi}{e^{\sigma h} \sin (\omega h)\left(\sigma^{2}+\omega^{2}\right)^{2}} .
\end{gathered}
$$

Here, $n=0, \pm 1, \pm 2, \ldots$, and $\Psi$ and $\Phi$ are defined as

$$
\begin{aligned}
\Psi= & 2 \omega \sigma\left(k_{p_{1}}-\left(\sigma^{2}+\omega^{2}\right)^{2}\right) \\
& -k_{r_{1}} e^{\tau \sigma}\left(2 \sigma \omega \cos (\omega \tau)-\left(\sigma^{2}-\omega^{2}\right) \sin (\omega \tau)\right), \\
\Phi= & \left(\sigma^{2}-\omega^{2}\right)\left(\sigma^{2} \omega^{2}+k_{p_{1}}-k_{r_{1}} e^{\tau \sigma} \cos (\omega \tau)\right) \\
& +k_{p_{2}}\left(\sigma^{2}+\omega^{2}\right)^{2}-2 k_{r_{1}} \sigma \omega e^{\tau \sigma} \sin (\omega \tau)+\left(\sigma^{6}-\omega^{6}\right) .
\end{aligned}
$$

The methodology to graphically determine the $\sigma$-stability boundaries (31)-(33) is as follows:

(1) Propose an initial value of $\sigma \in \mathbb{R}^{+}$close to zero, denoted by $\sigma_{0}$

(2) For the critical frequencies $s=0$ : from (31), graph the parametric boundaries for $k_{r_{2}}(h)$ on the parametric space $\left(h, k_{r_{2}}\right)$ considering $h \in(0, \bar{h}], \bar{h} \in \mathbb{R}^{+}$, and $\sigma_{0}$

(3) For the critical frequencies $s=i \omega$ :

(a) From (32), calculate $h$ considering $\omega \in(0, \bar{\omega}]$, $\bar{\omega} \in \mathbb{R}^{+}$, and $\sigma_{0}$

(b) From (33), compute $k_{r_{2}}$ considering $\omega \in(0, \bar{\omega}]$, $\sigma_{0}$, and $h$ (from (a)) (c) Plot the values of $h$ and $k_{r_{2}}$, obtained in the previous steps, on the parametric space $\left(h, k_{r_{2}}\right)$

(4) Detect the closed regions/curves in the parametric plane $\left(h, k_{r_{2}}\right)$ and discard the unstable ones; the remaining regions are considered as the $\sigma$-stability regions of (28)

(5) Select $\sigma_{1}>\sigma_{0}$

(6) Repeat steps $2-5$, considering $\sigma_{1}$, until the $\sigma$-stability regions collapse in a single point

Proposition 1 and Corollary 1 provide conditions to determine the $\sigma$-stability boundaries in the parametric plane $\left(h, k_{r_{2}}\right)$ of quasi-polynomial (28). Next, the main result of the contribution is presented, where analytic expressions relative to the tuning of the controller gains $h^{*}$ and $k_{r_{2}}^{*}$ to reach the maximal decay rate $\sigma^{*}$ are obtained. The result is based on the fact that when the maximal decay rate $\sigma^{*}$ is reached, there exist three dominant roots of the quasi-polynomial (28) in $s=-\sigma$; this phenomenon occurs when the concentric $\sigma$-stability regions collapse in a single point, denoted by $\left(h^{*}, k_{r_{2}}^{*}\right)$, as $\sigma$ increases. The value of $\sigma$, when the collapse occurs, determines the maximal decay rate $\sigma^{*}$; see $[25,30]$. Then, the analytical equations are determined to obtain the parameters $h^{*}$ and $k_{r_{2}}^{*}$, considering that there are three dominant roots on $\sigma^{*}$.

Proposition 2. Let us consider the closed-loop system (25). Then, the quasi-polynomial (28) has a root of multiplicity at least three on $s=-\sigma^{*}$ if $k_{r_{2}}^{*}$ and $h^{*}$ satisfy the following equations:

$$
\begin{aligned}
& h^{*}=h\left(\sigma^{*}\right)=\left.\left[\frac{(\partial / \partial s) P_{\sigma_{3}}(s)}{P_{\sigma_{3}}(0)}-\frac{(\partial / \partial s)\left(\Gamma\left(s, \sigma^{*}\right)\right)}{\Gamma\left(0, \sigma^{*}\right)}\right]\right|_{s=0}, \\
& k_{r_{2}}^{*}=k_{r_{2}}\left(\sigma^{*}, h^{*}\right)=\frac{-\left.(\partial / \partial s)\left(\Gamma\left(s, \sigma^{*}\right)\right)\right|_{s=0}}{\mathrm{e}^{\sigma^{*} h^{*}}\left(\left.(\partial / \partial s) P_{\sigma_{3}}(s)\right|_{s=0}-h^{*} P_{\sigma_{3}}(0)\right)},
\end{aligned}
$$

and $\sigma^{*}$ is the smallest positive real root of

$$
\begin{aligned}
& f\left(\sigma, k_{p_{1}}, k_{p_{2}}, k_{r_{1}}, \tau\right) \\
& =\left.P_{\sigma_{3}}(0)^{2}\left[\Gamma(0, \sigma) \frac{\partial^{2}}{\partial s^{2}}\left(\Gamma(s, \sigma)-\frac{\partial}{\partial s} \Gamma(s, \sigma)^{2}\right)\right]\right|_{s=0} \\
& \quad+\left.\Gamma(0, \sigma)^{2}\left[\left(\frac{\partial}{\partial s} P_{\sigma_{3}}(s)\right)^{2}-P_{\sigma_{3}}(0) \frac{\partial^{2}}{\partial s^{2}} P_{\sigma_{3}}(s)\right]\right|_{s=0} .
\end{aligned}
$$

Proof. Clearly, quasi-polynomial (28) presents three dominant roots at the point $s=-\sigma$ if the quasi-polynomial (29) has three dominant roots at $s=0$. Thus, the conditions

$$
\begin{array}{r}
\left.\mathrm{Q}_{\sigma}(\cdot)\right|_{s=0}=0, \\
\left.\frac{\mathrm{d}}{\mathrm{d} s} \mathrm{Q}_{\sigma}(\cdot)\right|_{s=0}=0, \\
\left.\frac{\mathrm{d}^{2}}{\mathrm{~d} s^{2}} \mathrm{Q}_{\sigma}(\cdot)\right|_{s=0}=0,
\end{array}
$$


must be satisfied; consequently,

$$
0=\Gamma(0, \sigma)+P_{\sigma_{3}}(0) k_{r_{2}} e^{\sigma h},
$$

$0=\left.\left(\frac{\partial}{\partial s} \Gamma(s, \sigma)\right)\right|_{s=0}+\left.k_{r_{2}} \mathrm{e}^{\sigma h}\left(\frac{\partial}{\partial s} P_{\sigma_{3}}(s) \mathrm{e}^{-s h}\right)\right|_{s=0}$,

$0=\left.\left(\frac{\partial^{2}}{\partial s^{2}} \Gamma(s, \sigma)\right)\right|_{s=0}+\left.k_{r_{2}} \mathrm{e}^{\sigma h}\left(\frac{\partial^{2}}{\partial s^{2}} P_{\sigma_{3}}(s) \mathrm{e}^{-s h}\right)\right|_{s=0}$.

The first two (44) and (45) can be easily verified. First, expression (45) follows directly from (49). Now, to obtain (44), from (48) and (49), it follows that

$$
0=\left.\left(\frac{\partial}{\partial s} \Gamma(s, \sigma)\right)\right|_{s=0}-\left.\frac{\Gamma(0, \sigma)}{P_{\sigma_{3}}(0)} \frac{\partial}{\partial s} P_{\sigma_{3}}(s)\right|_{s=0}+h \Gamma(0, \sigma),
$$

and we get (44):

$$
h=\left.\left[\frac{(\partial / \partial s) P_{\sigma_{3}}(s)}{P_{\sigma_{3}}(0)}-\frac{(\partial / \partial s)(\Gamma(s, \sigma))}{\Gamma(0, \sigma)}\right]\right|_{s=0} .
$$

To address (46), from (48) and (50), it follows that

$$
\begin{aligned}
0= & \left.\left(\frac{\partial^{2}}{\partial s^{2}} \Gamma(s, \sigma)\right)\right|_{s=0}+\left.k_{r_{2}} \mathrm{e}^{\sigma h} \frac{\partial^{2}}{\partial s^{2}} P_{\sigma_{3}}(s) \mathrm{e}^{-s h}\right|_{s=0} \\
= & \left.\left(\frac{\partial^{2}}{\partial s^{2}} \Gamma(s, \sigma)\right)\right|_{s=0}-\left.\frac{\Gamma(0, \sigma)}{P_{\sigma_{3}}(0)} \frac{\partial^{2}}{\partial s^{2}} P_{\sigma_{3}}(s)\right|_{s=0} \\
& +\left.2 h \frac{\Gamma(0, \sigma)}{P_{\sigma_{3}}(0)} \frac{\partial}{\partial s} P_{\sigma_{3}}(s)\right|_{s=0}-h^{2} \Gamma(0, \sigma) .
\end{aligned}
$$

Substituting (52) in the above equation yields

$$
\begin{aligned}
0= & \left.P_{\sigma_{3}}(0)^{2}\left[\Gamma(0, \sigma) \frac{\partial^{2}}{\partial s^{2}} \Gamma(s, \sigma)-\left(\frac{\partial}{\partial s} \Gamma(s, \sigma)\right)^{2}\right]\right|_{s=0} \\
& +\left.\Gamma(0, \sigma)^{2}\left[\left(\frac{\partial}{\partial s} P_{\sigma_{3}}(s)\right)^{2}-P_{\sigma_{3}}(0) \frac{\partial^{2}}{\partial s^{2}} P_{\sigma_{3}}(s)\right]\right|_{s=0},
\end{aligned}
$$

which ends proof.

In the following corollary, conditions (44)-(46) are written particularly for the quasi-polynomial (27), where $P_{1}\left(s, k_{p_{1,2}}\right)=s^{4}+k_{p_{2}} s^{2}+k_{p_{1}}, P_{2}\left(s, k_{r_{1}}\right)=-k_{r_{1}}$, and $P_{3}(s)=$ $-s^{2}$.

Corollary 2. Consider the quasi-polynomial of the form (27); then, the controller gains $h^{*}, k_{r_{2}}^{*}$ and the function $f(\sigma, \cdot)$ given by equations (44)-(46) look as

$$
\begin{aligned}
f\left(\sigma, k_{p_{1,2}}, k_{r_{1}}, \tau\right)= & \sigma^{4}\left(2 \sigma\left(k_{p_{2}}-2 \sigma^{2}\right)-k_{r_{1}} \tau e^{\sigma \tau}\right)^{2} \\
& +\sigma^{2} \kappa\left[\sigma^{2}\left(2 k_{p_{2}}+12 \sigma^{2}-k_{r_{1}} \tau^{2} e^{\sigma \tau}\right)\right] 2 \sigma^{2} \kappa^{2},
\end{aligned}
$$

where $\kappa=k_{p_{1}}-k_{r_{1}} e^{\sigma \tau}+\sigma^{2}\left(\sigma^{2}+k_{p_{2}}\right)$ and

$$
\begin{aligned}
h^{*}\left(\sigma, k_{p_{1,2}}, k_{r_{1}}, \tau\right) & =\frac{2 \sigma \mu_{1}-k_{r_{1}} \tau e^{\sigma \tau}}{k_{p_{1}}-k_{r_{1}} e^{\sigma \tau}+\sigma^{2} \mu_{2}}-\frac{2}{\sigma}, \\
k_{r_{2}}^{*}\left(\sigma, k_{p_{1,2}}, k_{r_{1}}, h, \tau\right) & =e^{\sigma h} \frac{2 \sigma \mu_{1}-k_{r_{1}} \tau e^{\sigma \tau}}{\sigma(2+h \sigma)},
\end{aligned}
$$

with $\mu_{1}=k_{p_{2}}+2 \sigma^{2}$ and $\mu_{2}=\mu_{1}-\sigma^{2}$.

The conditions introduced in this section are only focused on the appropriate selection of the control parameters $\sigma^{*}, k_{r_{2}}^{*}$, and $h^{*}$ that guarantee reaching the maximal exponential decay rate, but there are no conditions involving the remaining control parameters. In the following, a simple approach for the approximate selection of the control parameters is presented. On the one hand, let us consider quasi-polynomial (27), where the exponential terms are estimated by its first-order Taylor series truncated expansion; that is,

$$
\begin{aligned}
Q\left(s, k_{p_{1,2}}, k_{r_{1,2}}, \tau, h\right)= & \left(s^{4}+k_{p_{2}} s^{2}+k_{p_{1}}\right)-k_{r_{1}}(1-s \tau) \\
& -k_{r_{2}} s^{2}(1-s h) \\
= & s^{4}+h k_{r_{2}} s^{3}+\left(k_{p_{2}}-k_{r_{2}}\right) s^{2}+\tau k_{r_{1}} s \\
& +\left(k_{p_{1}}-k_{r_{1}}\right) .
\end{aligned}
$$

Now, let us propose a Hurwitz stable polynomial of the following form:

$$
p(s)=\left(s^{2}+2 \xi_{c} \omega_{c} s+\omega_{c}^{2}\right)^{2},
$$

where $\xi_{c}, \omega_{c} \in \mathbb{R}^{+}$. Thus, the dynamics of the previous polynomial is matched with (57). Then, simple calculations show that the controller gain parameters $k_{p_{1}}, k_{p_{2}}, k_{r_{1}}$, and $\tau$ can be chosen according to the following conditions (for $\alpha_{1}>1$ and $\alpha_{2}>2$ ):

$$
\begin{aligned}
& k_{p_{1}}>\alpha_{1} \omega_{c}^{4}, \\
& k_{p_{2}}>\alpha_{2} \omega_{c}^{2}\left(1+2 \xi_{c}^{2}\right), \\
& k_{r_{1}}=k_{p_{1}}-\omega_{c}^{4}, \\
& \tau=\frac{4 \xi_{c} \omega_{c}^{3}}{k_{r_{1}}} .
\end{aligned}
$$

Remark 1. Due to the simple nature of conditions (54) and (60), they must be considered only as a starting point in the process of tuning the delayed control law (24) and subsequently adjusted according to the performance of the system.

\section{Cases of Study}

5.1. Rotatory Flexible Link. The rotatory flexible link platform consists of a rotating base driven by a belt-pulley mechanism actuated by a DC motor, a link is attached to the rotating base by two springs of identical nature, which results in a flexible joint. The schematic of such a manipulator is presented in Figure 2. The variables $\theta_{1}(t)$ and $\theta_{2}(t)$ denote 


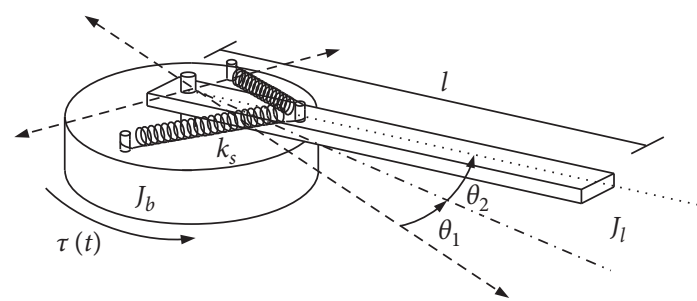

FIGURE 2: Schematics of the rotatory flexible link.

the angular positions of the rotating base and the link, respectively, $l$ represents the length of the link, $J_{b}$ stands for the inertia of the rotating base, and $J_{l}$ is the moment of inertia of the link. Finally, $k_{s}$ denotes the stiffness of the spring and $u(t)$ is the torque input applied to the system.

The problem formulation is stated as follows. For the rotatory flexible link, a smooth rest to rest angular position reference trajectory for the rotating base is demanded while the nonactuated link is desired to remain in a vicinity zero position, avoiding oscillations during the tracking maneuver.

5.1.1. The Dynamic Model. Following the methodology presented in Section 3 the Euler-Lagrange formalism (11) is used to represent the mathematical model of the rotatory flexible link system, where the viscous friction is neglected and the unmodeled nonlinear terms are ignored:

$$
M(q(t)) \ddot{q}(t)+C(q(t), \dot{q}(t)) \dot{q}(t)+g(q(t))=P \tau(t),
$$

with

$$
\begin{aligned}
M(q) & =\left[\begin{array}{cc}
J_{b}+J_{l} & J_{l} \\
J_{l} & J_{l}
\end{array}\right], \\
C(q, \dot{q}) & =\left[\begin{array}{ll}
0 & 0 \\
0 & 0
\end{array}\right], \\
g(q) & =\left[\begin{array}{c}
0 \\
k_{s} \theta_{2}(t)
\end{array}\right], \\
q(t) & =\left[\begin{array}{ll}
\theta_{1}(t) & \theta_{2}(t)
\end{array}\right]^{\top}, \\
P & =\left[\begin{array}{ll}
1 & 0
\end{array}\right]^{\top},
\end{aligned}
$$

or in an equivalent form:

$$
\begin{aligned}
& \ddot{\theta}_{1}(t)=\frac{k_{s}}{J_{b}} \theta_{2}(t)+\frac{\tau(t)}{J_{b}}, \\
& \ddot{\theta}_{2}(t)=-k_{s}\left(\frac{J_{b}+J_{l}}{J_{b} J_{l}}\right) \theta_{2}(t)-\frac{\tau(t)}{J_{b}} .
\end{aligned}
$$

5.1.2. Cascade Structure Representation. Let us introduce the state vector:

$$
\begin{aligned}
x(t) & =\left[\begin{array}{llll}
x_{1}(t) & x_{2}(t) & x_{3}(t) & x_{4}(t)
\end{array}\right]^{\top} \\
& =\left[\begin{array}{llll}
\theta_{1}(t) & \dot{\theta}_{1}(t) & \theta_{2}(t) & \dot{\theta}_{2}(t)
\end{array}\right]^{\top} .
\end{aligned}
$$

Then, the applied torque can be expressed as $\tau(t)=N \tau_{m}$, where $\tau_{m}=\left(k_{\tau} / R_{m}\right) V(t)$ is the torque in terms of the input voltage, $N$ represents the gear ratio, $k_{\tau}$ is the torque constant, and $R_{m}$ denotes the motor armature resistance. Since (63) is linear, it can be rewritten in space state representation (24) as follows:

$$
\dot{x}(t)=A x(t)+b V(t),
$$

$$
\begin{aligned}
A & =\left[\begin{array}{cccc}
0 & 1 & 0 & 0 \\
0 & 0 & \frac{k_{s}}{J_{b}} & 0 \\
0 & 0 & 0 & 1 \\
0 & 0 & -\left(\frac{J_{l}+J_{b}}{J_{l} J_{b}}\right) k_{s} & 0
\end{array}\right], \\
b & =\left[\begin{array}{c}
\frac{N k_{\tau}}{R_{m} J_{b}} \\
0 \\
-\frac{N k_{\tau}}{R_{m} J_{b}}
\end{array}\right] .
\end{aligned}
$$

Let us define the Kalman controllability matrix as in (5). Direct calculations show that the pair $(A, b)$ is controllable and consequently, by Definition 2, it is flat. The flat output can be computed according to (16), where by defining $\varepsilon=\left(R_{m} J_{b} J_{l}\right) /\left(k_{\tau} k_{s} N\right)$, the flat output $y_{f}(t)$ is expressed as

$$
y_{f}(t)=x_{1}(t)+x_{3}(t)
$$

The flat output time derivatives are

$$
\begin{aligned}
y_{f}(t) & =x_{1}(t)+x_{2}(t), \\
\dot{y}_{f}(t) & =x_{2}(t)+x_{4}(t), \\
\ddot{y}_{f}(t) & =-\frac{k_{s}}{J_{l}} x_{3}(t), \\
y_{f}^{(3)}(t) & =-\frac{k_{s}}{J_{l}} x_{4}(t), \\
y_{f}^{(4)}(t) & =\frac{k_{s}^{2}\left(J_{l}+J_{b}\right)}{J_{l}^{2} J_{b}} x_{3}(t)+\frac{k_{\tau} k_{s} N}{R_{m} J_{b} J_{l}} V(t) .
\end{aligned}
$$

Let us define the output error as in (20); then, its dynamics are

$$
\begin{aligned}
& \dot{e}_{1}(t)=e_{2}(t), \\
& \dot{e}_{2}(t)=e_{3}(t), \\
& \dot{e}_{3}(t)=e_{4}(t), \\
& \dot{e}_{4}(t)=\frac{k_{s}^{2}\left(J_{l}+J_{b}\right)}{J_{l}^{2} J_{b}} x_{3}(t)+\frac{k_{\tau} k_{s} N}{R_{m} J_{b} J_{l}} V(t)-y_{f}^{*(4)}(t),
\end{aligned}
$$


where the auxiliary control $V(t)$ is proposed as

$$
\begin{aligned}
V(t) & =a_{1}\left[u(t)-\frac{k_{s}^{2}\left(J_{l}+J_{b}\right)}{J_{l}^{2} J_{b}} x_{3}(t)+y_{f}^{*(4)}(t)\right], \\
a_{1} & =\frac{R_{m} J_{b} J_{l}}{k_{\tau} k_{s} N} .
\end{aligned}
$$

5.1.3. Delayed Control Law. If the following delayed controller, $u(t)={ }_{u}(t)$ is considered:

$$
u_{D}(t)=-k_{p_{1}} e_{1}(t)+k_{r_{1}} e_{1}(t-\tau)-k_{p_{2}} e_{3}(t)+k_{r_{2}} e_{3}(t-h) .
$$

The chain of integrators representation (25) is obtained.

Remark 2. It is worth noting that both the auxiliary control $V(t)$ and the delayed controller $u_{D}(t)$ depend completely on the measurable variables that correspond to angular positions $x_{1}(t)=\theta_{1}(t)$ and $x_{3}(t)=\theta_{2}(t)$.

5.1.4. Feedback State Control Law. In order to compare the proposed delayed controller with a classical scheme, a feedback state control is designed as follows:

$$
u_{\mathrm{FS}}(t)=-\kappa_{1} e_{1}(t)-\kappa_{2} e_{2}-\kappa_{3} e_{3}(t)-\kappa_{4} e_{4}(t),
$$

where the set of gains $\left[\kappa_{1}, \kappa_{2}, \kappa_{3}, \kappa_{4}\right]$ is chosen as $\kappa_{1}=\omega^{4}$, $\kappa_{2}=4 \bar{\zeta} \omega^{3}, \kappa_{3}=4 \bar{\varsigma}^{2} \omega^{2}+2 \varpi^{2}$, and $\kappa_{4}=4 \bar{\zeta} \omega$.

Remark 3. Now, the auxiliary control $V(t)$ and the feedback state control law $u_{\mathrm{FS}}(t)$ depend on the complete vector state $x(t)$, due to the fact that the velocity states $x_{2}(t)=\dot{\theta}_{1}(t)$ and $x_{4}(t)=\dot{\theta}_{2}(t)$ are not available; then, a low pass filter with transfer function $G(s)=(200 s) /(s+200)$ is used to estimate the velocity using the measurable variables $x_{1}(t)=\theta_{1}(t)$ and $x_{3}(t)=\theta_{2}(t)$; this methodology allows us to reduce the noise generated by the estimation of the so-called "dirty derivative" [45].

5.1.5. Experimental Results. In Figure 3, the rotatory flexible link experimental platform is presented. The prototype consists of a DC motor NISCA model NC5475 attached to the rotating base by means of a belt-pulley system with a 16 : 1 ratio. The angular position of both the rotating base and the link is measured by means of incremental encoders with a resolution of 1000 counts per revolution. The data acquisition is carried out with a data card Sensoray, model 626. The data card acquires the signals from the optical incremental encoders and supplies the control voltages to the power amplifiers (Quanser amplifier model VoltPAQ-X2). The control scheme is implemented in the Matlab-Simulink platform with a sampling time of 0.001 [s].

The rotatory flexible link parameters are

$$
\begin{aligned}
& \text { Inertias: } J_{b}=0.0481 \quad\left[\mathrm{Kg}-\mathrm{m}^{2}\right] \text { and } J_{l}=0.0036 \\
& {\left[\mathrm{Kg}-\mathrm{m}^{2}\right] .} \\
& \text { Length of the link: } l=0.55[\mathrm{~m}] .
\end{aligned}
$$

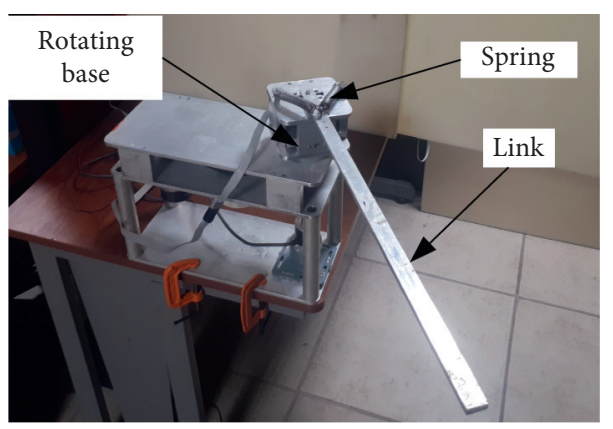

Figure 3: Rotatory flexible link prototype.

Stiffness of the spring: $k_{s}=4[\mathrm{~N}-\mathrm{m} / \mathrm{rad}]$. Armature resistance: $k_{\tau}=0.0724$ [ $\Omega$ ].

Torque constant: $\tau_{m}=2.983[\Omega]$.

Mechanical advantage: $N=16$.

In this experiment, the tracking trajectory problem is addressed. The initial conditions are $x(0)=0$. The desired trajectory $y_{f}^{*}(t)$ consists of a path initialized at $y_{f}^{*}(0)=0[\mathrm{rad}]$. After two seconds in this position, the mechanism moves, in an interval of $0.7[\mathrm{~s}]$, to $y_{f}^{*}(2)=\pi / 2[\mathrm{rad}]$ where it stands still for $7.3[\mathrm{~s}]$. Finally, the reference path returns to its initial condition in an interval of $0.7[\mathrm{~s}]$.

The parameters of the controller were selected as follows. According to (38) and (39), the following values were proposed $\omega_{c}=52$ and $\xi_{c}=1.2 ;$ thus, $k_{p_{1}}=29246464$, $k_{p_{2}}=33572.864, k_{r_{1}}=21934848$, and $\tau=0.02$. Now, the conditions presented in Corollary 4 lead to $\sigma^{*}=66.65$ and consequently $k_{r_{2}}^{*}=18839.729$ and $h^{*}=0.00476$.

The $\sigma$-stability boundaries were calculated following Corollary 1, which is illustrated in Figure 4. The red mark symbolizes the maximal achievable decay rate $\sigma^{*}$ and, as it can be seen, represents the point where all the $\sigma$-stable regions collapse.

Figure 5 depicts the rightmost root locus of the closedloop system, where it can be appreciated that a triple real dominant root is located at the point $\sigma^{*}=66.65$ as stated in Proposition 3.

The set of gains for the feedback state controller were chosen as $\Phi=52$ and $\bar{\varsigma}=1.2$. The main challenge when controlling the rotatory flexible link platform is to avoid the oscillations that appear intrinsically due to the flexible nature of the system. In Figure 6, the rotatory flexible link tracking trajectory task under the control actions of the proposed cascade PR scheme and feedback state is presented. The desired trajectory $y_{f}^{*}(t)$ is represented by a black line, while the flat output $y_{\mathrm{fD}}(t)$ with the delayed controller is depicted in blue and the $y_{\mathrm{fFS}}(t)$ with the feedback is represented by a red line. It can be seen that the tracking task is carried out satisfactorily by both controllers, avoiding oscillations and reaching the reference without overshooting. The tracking errors are detailed in Figure 7, noticing that both errors $e_{D}$ and $e_{\mathrm{FS}}$ are restricted to the interval $[-0.075,0.075][\mathrm{rad}]$ with similar performance. Figures 8 and 9 represent the evolution of the angular positions $\theta_{1}$ and $\theta_{2}$ 


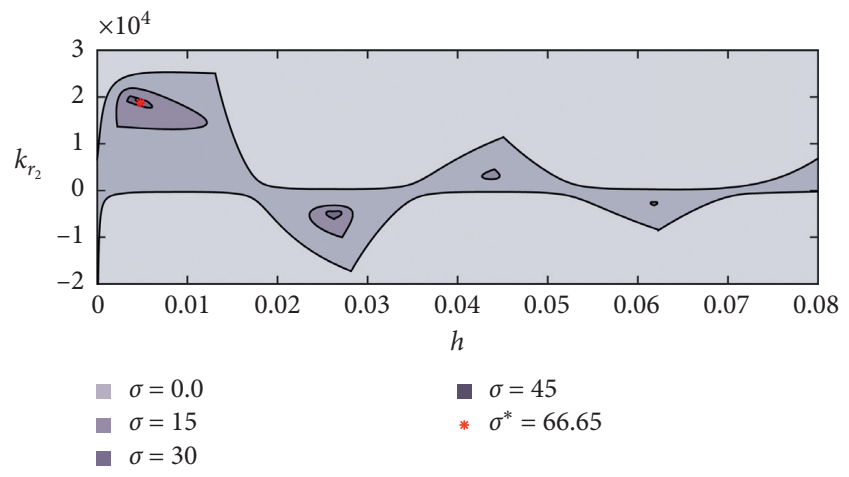

Figure 4: $\sigma$-stability boundaries.

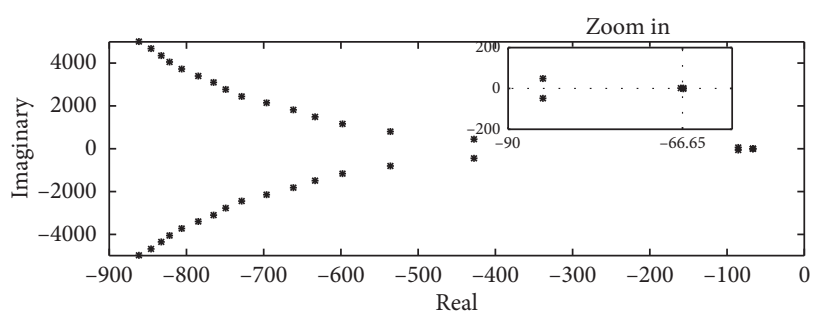

Figure 5: Root locus.

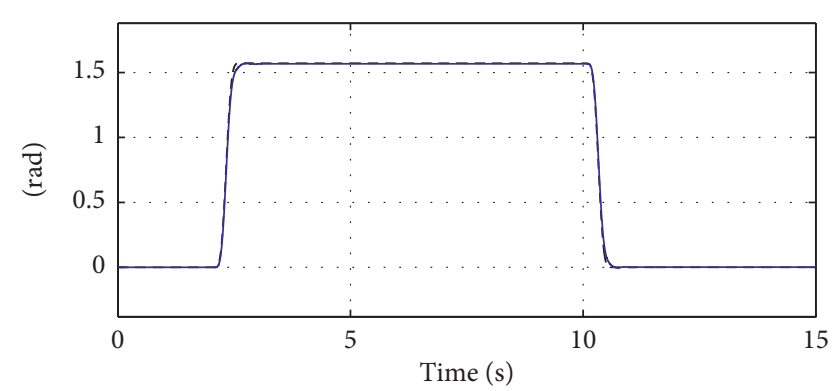

$$
\begin{aligned}
& --y_{f}^{*}(t) \\
& -y_{f D}(t)
\end{aligned}
$$

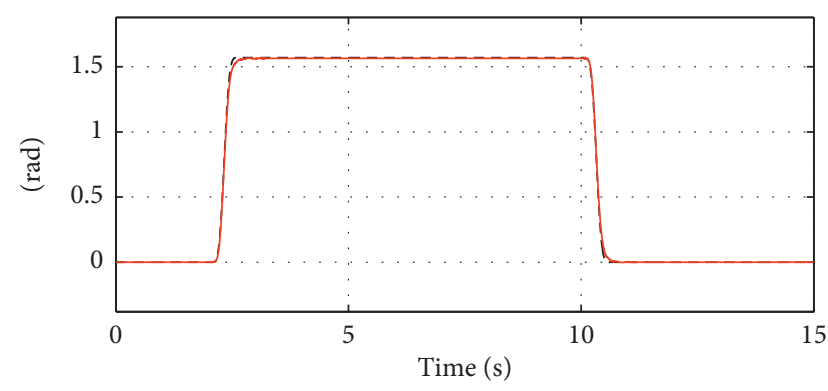

$$
\begin{aligned}
& ---y_{f}^{*}(t) \\
& -y_{f F S}(t)
\end{aligned}
$$

FIGURE 6: Trajectory tracking results.

when the system follows the reference trajectory. Figure 10 shows the control voltages of both schemes. Notice that the peak voltage $V_{D}$ does not exceed \pm 10 [Volts] but $V_{\mathrm{FS}}$ presents a larger amplitude. As a consequence of avoiding the use of the time derivatives in the proposed control scheme, the voltage $V_{D}$ signal appears relatively free of noise, as expected

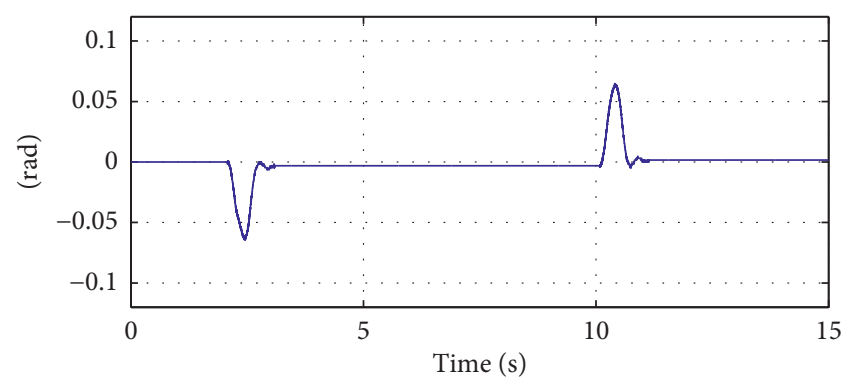

$-e_{D}(t)$

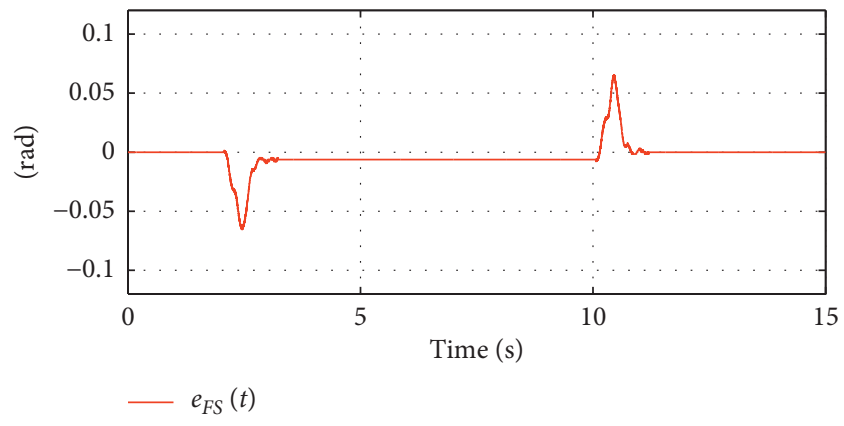

Figure 7: Tracking error.

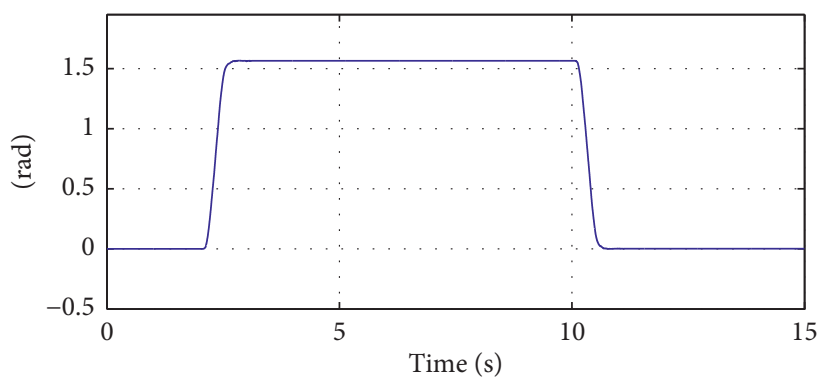

$-\theta_{1 D}(t)$

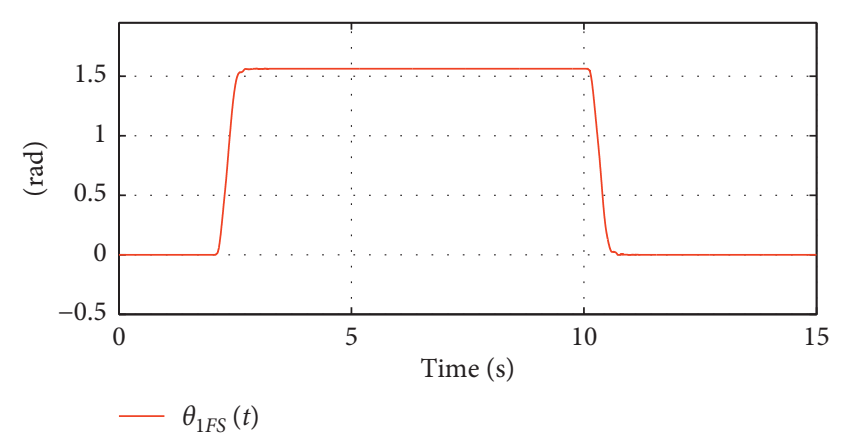

FIgURE 8: $\theta_{1}$ evolution.

but $V_{\mathrm{FS}}$ presents high-frequency components; it can be corroborated in Figure 11 where a Power Spectrum Density of control signals $V_{D}$ and $V_{\mathrm{FS}}$ is presented. Here, it can be observed that $F S_{\mathrm{PSD}}$ presents more frequency components in comparison with $D_{\text {PSD }}$. Finally, the performance of the system is evaluated by means of a quadratic index of the applied voltage (see Figure 12) where it can be noticed that $V_{\mathrm{FS}}$ consumes more energy. 


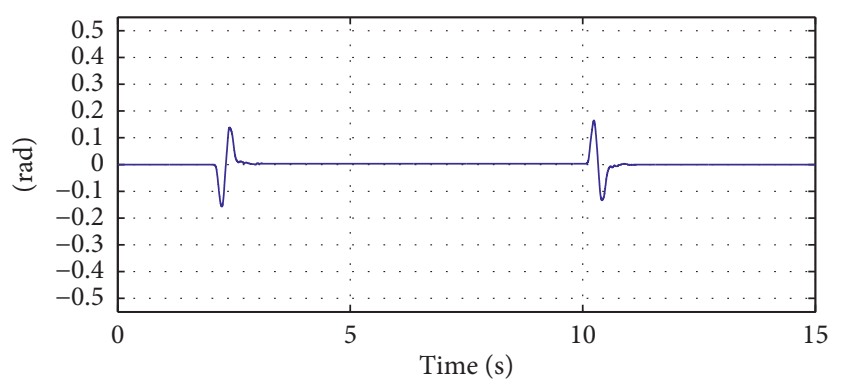

$-\theta_{2 D}(t)$

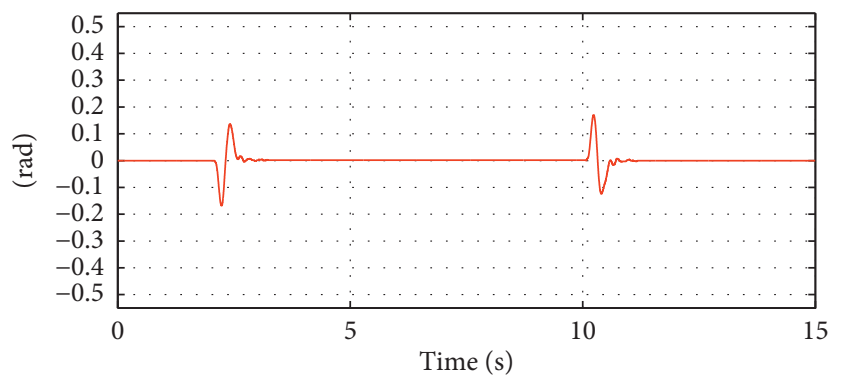

$-\theta_{2 F S}(t)$

Figure 9: $\theta_{2}$ evolution.

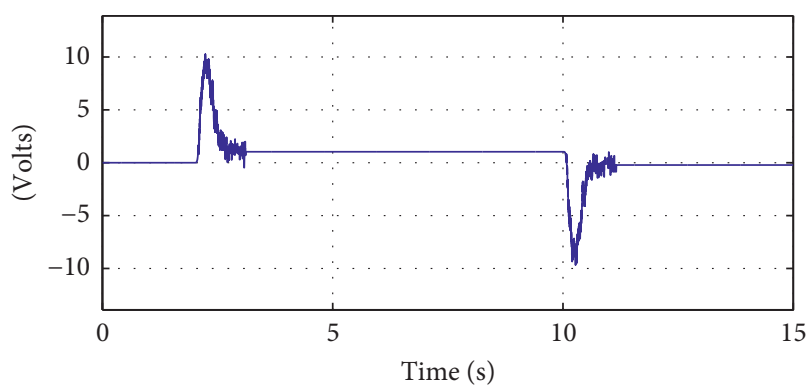

$-V_{D}(t)$

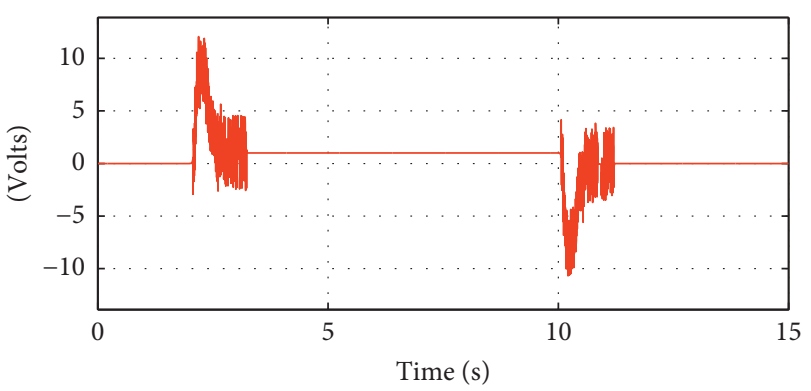

$-V_{F S}(t)$

FIgURE 10: Control input voltage.

5.2. Pendubot System. The pendubot consists of a planar double inverted pendulum, whose schematic is presented in Figure 13. The first link is driven by a DC motor while the second link is an underactuated simple pendulum. The variables $\theta_{1}$ and $\theta_{2}$ denote the angular positions of the links, $u$ represents the control torque input, and the parameters $m_{1}$
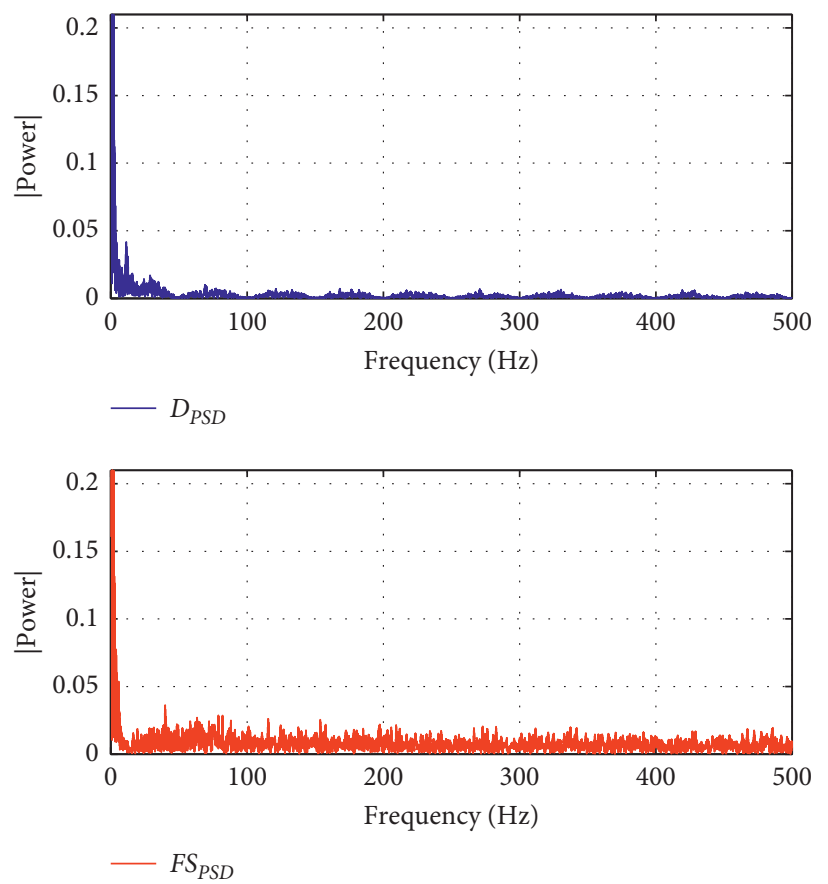

Figure 11: Power spectrum density.

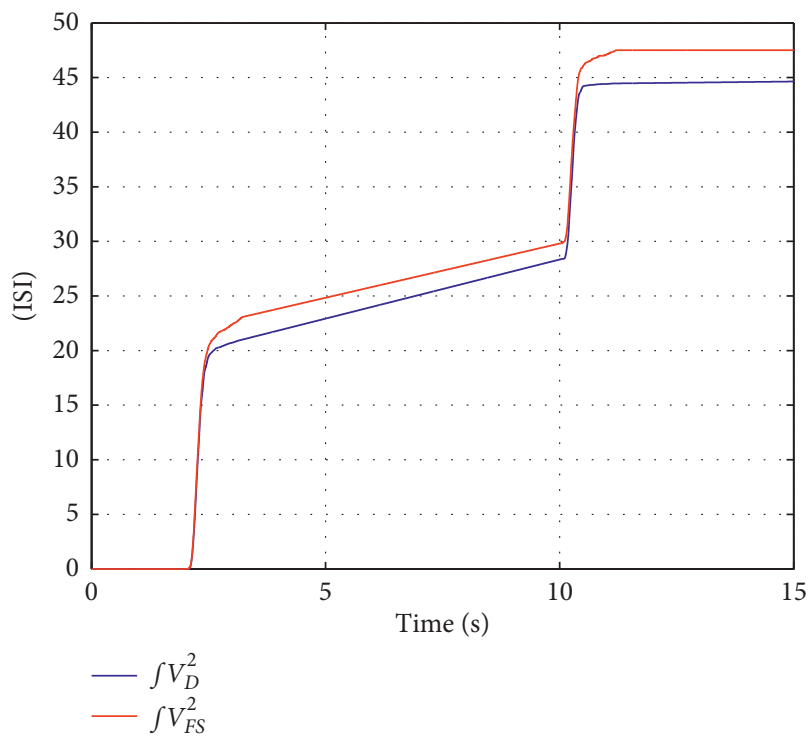

FIgURE 12: Performance index of the controller.

and $m_{2}$ denote the masses of the links. The lengths of the links are represented as $l_{1}$ and $l_{2}$, while $l_{c_{1}}$ and $l_{c_{2}}$ are the distances to the center of the masses. Finally, $I_{1}$ and $I_{2}$ denote the inertias of the links.

The problem formulation is given as follows: a smooth rest to rest angular position reference trajectory for the first link is demanded, while the nonactuated second link is desired to remain around its unstable vertical position, without falling during the entire tracking maneuver or moving away from the equilibrium point. 


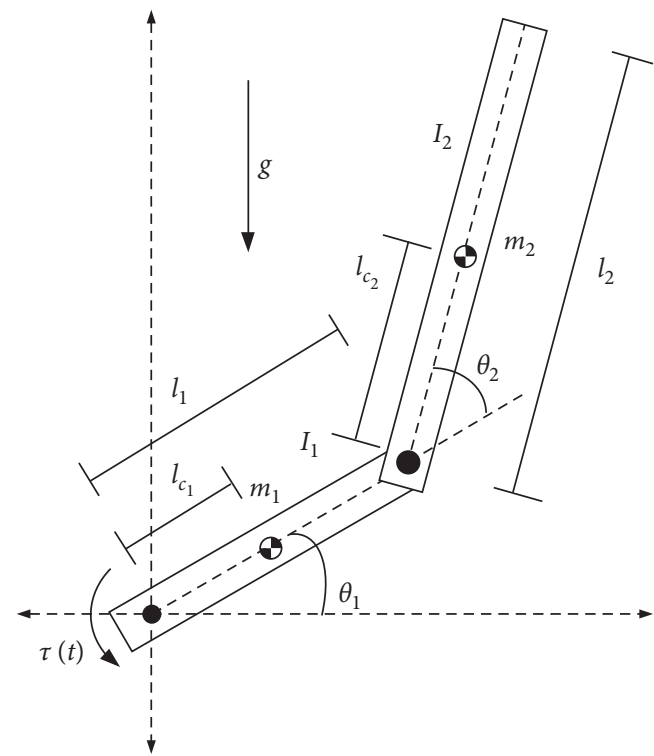

FIGURE 13: Schematics of the pendubot system.

5.2.1. The Nonlinear Dynamic Model. As carried out for the flexible link system, the Euler-Lagrange formalism is used to represent the dynamic model of the considered system:

$$
M(q(t)) \ddot{q}(t)+C(q(t), \dot{q}(t)) q(t)+g(q(t))=P \tau(t),
$$

with $q(t)=\left[\begin{array}{ll}\theta_{1}(t) & \theta_{2}(t)\end{array}\right]^{\top}$ and $P=\left[\begin{array}{ll}1 & 0\end{array}\right]^{\top}$. Here,

$$
\begin{aligned}
M(q) & =\left[\begin{array}{cc}
\beta_{1}+\beta_{2}+2 \beta_{3} \cos \left(\theta_{2}(t)\right) & M_{1,2}(\theta) \\
\beta_{2}+\beta_{3} \cos \left(\theta_{2}(t)\right) & \beta_{2}
\end{array}\right], \\
C(q, \dot{q}) & =\left[\begin{array}{cc}
-\beta_{3} \dot{\theta}_{1}(t) \sin \left(\theta_{2}(t)\right) & \beta_{3} C_{1,2}(\theta) \\
\beta_{3} \dot{\theta}_{1}(t) \sin \left(\theta_{2}(t)\right) & 0
\end{array}\right], \\
g(q) & =\left[\begin{array}{c}
\beta_{4} g \cos \left(\theta_{1}(t)\right)+\beta_{5} g \cos \left(\theta_{1}(t)+\theta_{2}(t)\right) \\
\beta_{5} g \cos \left(\theta_{1}(t)+\theta_{2}(t)\right)
\end{array}\right],
\end{aligned}
$$

where

$$
\begin{aligned}
\beta_{1} & =m_{1} l_{c_{1}}^{2}+m_{2} l_{c_{2}}^{2}+I_{1}, \\
\beta_{2} & =m_{2} l_{c_{2}}^{2}+I_{2}, \\
\beta_{3} & =m_{2} l_{1} l_{c_{2}}, \\
\beta_{4} & =m_{1} l_{c_{1}}+m_{1} l_{1}, \beta_{5}=m_{2} l_{c_{2}}, \\
M_{1,2}(\theta) & =\beta_{2}+\beta_{3} \cos \left(\theta_{2}(t)\right), \\
C_{1,2}(\theta) & =\left(\dot{\theta}_{1}(t)+\dot{\theta}_{2}(t)\right) \sin \left(\theta_{2}(t)\right) .
\end{aligned}
$$

5.2.2. Cascade Structure Representation. In this section, the procedure presented in Section 3 is applied. The state vector is defined as

$$
\begin{aligned}
x(t) & =\left[\begin{array}{llll}
x_{1}(t) & x_{2}(t) & x_{3}(t) & x_{4}(t)
\end{array}\right]^{\top} \\
& =\left[\begin{array}{llll}
\theta_{1}(t) & \dot{\theta}_{1}(t) & \theta_{2}(t) & \dot{\theta}_{2}(t)
\end{array}\right]^{\top} .
\end{aligned}
$$

The applied torque can be expressed as $\tau(t)=\left(k_{\tau} / R_{m}\right) V(t)$ in terms of the input voltage, where $k_{\tau}$ is the torque constant and $R_{m}$ represents the motor armature resistance. For $V=0$, the considered equilibrium point $\bar{x}(t)$ is

$$
\bar{x}(t)=\left[\begin{array}{llll}
\frac{\pi}{2} & 0 & 0 & 0
\end{array}\right]^{\top}
$$

Then, the tangent linearization looks as

$$
\dot{x}_{\delta}(t)=A x_{\delta}(t)+b V(t)
$$

where $x_{\delta}(t)=\left[\begin{array}{llll}x_{1}(t)-\pi 2 & x_{2}(t) & x_{3}(t) & x_{4}(t)\end{array}\right]^{\top}$,

$$
\begin{aligned}
& A=\left[\begin{array}{cccc}
0 & 1 & 0 & 0 \\
\frac{\left(\beta_{3} \beta_{5}-\beta_{2} \beta_{4}\right) g}{\beta_{3}^{2}-\beta_{1} \beta_{2}} & 0 & \frac{\beta_{3} \beta_{5} g}{\beta_{3}^{2}-\beta_{1} \beta_{2}} & 0 \\
0 & 0 & 0 & 1 \\
\frac{\left(\left(\beta_{2}+\beta_{3}\right) \beta_{4}-\left(\beta_{1}+\beta_{3}\right) \beta_{5}\right) g}{\beta_{3}^{2}-\beta_{1} \beta_{2}} & 0-\frac{\left(\beta_{1}+\beta_{3}\right) \beta_{5} g}{\beta_{3}^{2}-\beta_{1} \beta_{2}} & 0
\end{array}\right], \\
& b=\left[\begin{array}{cccc}
0-\frac{k_{\tau} \beta_{2}}{R_{m}\left(\beta_{3}^{2}-\beta_{1} \beta_{2}\right)} & 0 & \frac{\left.k_{2}+\beta_{3}\right)}{R_{m}\left(\beta_{3}^{2}-\beta_{1} \beta_{2}\right)}
\end{array}\right]^{T} .
\end{aligned}
$$

By defining the Kalman controllability matrix as $K_{c}=\left[\begin{array}{llll}b & A b & A^{2} b & A^{3} b\end{array}\right]$, the pair $(A, b)$ is controllable since $K_{c}$ has full rank. Then, according to Definition 2, it implies that system (42) is flat, and the corresponding flat output is computed as

$$
y_{f}(t)=\varepsilon\left[\begin{array}{llll}
0 & 0 & 0 & 1
\end{array}\right] K_{c}^{-1} x_{\delta}(t)=\frac{\beta_{2}+\beta_{3}}{\beta_{2}} x_{\delta 1}(t)+x_{\delta 3}(t)
$$

Here, $\varepsilon$ is selected as $\varepsilon=\left(R_{m} \beta_{2}\left(\beta_{3}^{2}-\beta_{1} \beta_{2}\right)\right) /\left(k_{\tau} \beta_{3} \beta_{5} g\right)$. Now, by straightforward calculations, the flat output time derivatives (10) are given by

$$
\begin{aligned}
y_{f}(t) & =\frac{\beta_{2}+\beta_{3}}{\beta_{2}} x_{\delta 1}(t)+x_{\delta 3}(t), \\
\dot{y}_{f}(t) & =\frac{\beta_{2}+\beta_{3}}{\beta_{2}} x_{\delta 2}(t)+x_{\delta 4}(t), \\
\ddot{y}_{f}(t) & =\frac{g \beta_{5}}{\beta_{2}}\left(x_{\delta 1}(t)+x_{\delta 3}(t)\right), \\
y_{f}^{(3)}(t) & =\frac{g \beta_{5}}{\beta_{2}}\left(x_{\delta 2}(t)+x_{\delta 4}(t)\right), \\
y_{f}^{(4)}(t) & =\rho_{1} x_{\delta 1}(t)-\rho_{2}\left(x_{\delta 1}(t)+x_{\delta 3}(t)\right)+\rho_{3} V(t),
\end{aligned}
$$

with 


$$
\begin{aligned}
& \rho_{1}=\frac{\left(\beta_{3} \beta_{4} \beta_{5}\right) g^{2}}{\beta_{2}\left(\beta_{3}^{2}-\beta_{1} \beta_{2}\right)}, \\
& \rho_{2}=\frac{\beta_{1} \beta_{5}^{2} g^{2}}{\beta_{2}\left(\beta_{3}^{2}-\beta_{1} \beta_{2}\right)}, \\
& \rho_{3}=\frac{k_{\tau} \beta_{3} \beta_{5} g}{R_{m} \beta_{2}\left(\beta_{3}^{2}-\beta_{1} \beta_{2}\right)} .
\end{aligned}
$$

By defining the output error as in (20), then, the set of error dynamics (21) are given as follows:

$$
\begin{aligned}
\dot{e}_{1}(t)= & e_{2}(t), \\
\dot{e}_{2}(t)= & e_{3}(t), \\
\dot{e}_{3}(t)= & e_{4}(t), \\
\dot{e}_{4}(t)= & \rho_{1} x_{\delta 1}(t)-\rho_{2}\left(x_{\delta 1}(t)+x_{\delta 3}(t)\right) \\
& +\rho_{3} u_{\delta 1}(t)-y_{f}^{*(4)}(t) .
\end{aligned}
$$

Now, by proposing the auxiliary control

$$
\begin{aligned}
V(t)= & \rho_{3}^{-1}\left[u(t)-\rho_{1} x_{\delta 1}(t)+\rho_{2}\left(x_{\delta 1}(t)+x_{\delta 3}(t)\right)\right] \\
& +\rho_{3}^{-1} y_{f}^{*(4)}(t),
\end{aligned}
$$

and by considering the delayed control law

$$
\begin{aligned}
u(t)= & u_{D}(t)=-k_{p_{1}} e_{1}(t)+k_{r_{1}} e_{1}(t-\tau)-k_{p_{2}} e_{3}(t) \\
& +k_{r_{2}} e_{3}(t-h),
\end{aligned}
$$

the desired chain of integrators representation (25) is obtained.

Remark 4. As in the previous experiment, the auxiliary control $V(t)$ and the delayed control law $u_{D}(t)$ depend completely on the measurable variables that correspond to the angular positions.

5.2.3. Feedback State Control Law. As in the previous test, feedback state control is designed in order to compare its performance with the proposed delayed controller:

$$
u_{\mathrm{FS}}(t)=-\kappa_{1} e_{1}(t)-\kappa_{2} e_{2}-\kappa_{3} e_{3}(t)-\kappa_{4} e_{4}(t),
$$

where the following set of gains $\left[\kappa_{1}, \kappa_{2}, \kappa_{3}, \kappa_{4}\right]$ is chosen as $\kappa_{1}=\omega^{4}, \kappa_{2}=4 \bar{\zeta} \omega^{3}, \kappa_{3}=4 \bar{\varsigma}^{2} \omega^{2}+2 \Theta^{2}$, and $\kappa_{4}=4 \bar{\zeta} \omega$.

Remark 5. The velocity states $x_{2}(t)$ and $x_{4}(t)$ are estimated using a low pass filter with transfer function $G(s)=(200 s / s+200)$.

5.2.4. Experimental Results. Figure 14 shows the experimental pendubot prototype. It consists of a DC motor NISCA model NC5475, which drives the first link. The angular position of both links is measured by means of incremental encoders with a resolution of 10000 counts per revolution. The same data acquisition model of the former example was used. The power amplifier consists of a Quanser

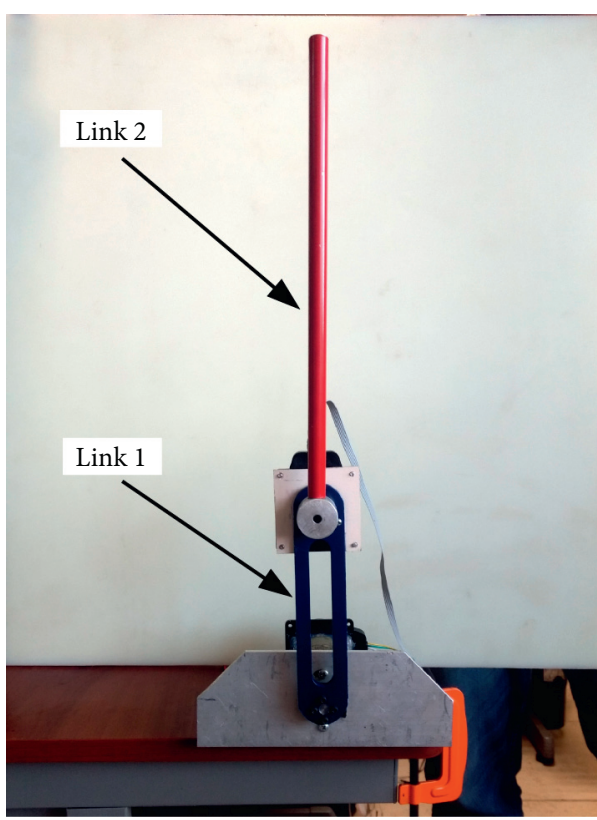

Figure 14: Pendubot prototype.

amplifier model VoltPAQ-X2. The control strategy was implemented in the Matlab-Simulink platform, and the sampling time was set to be $0.001[\mathrm{~s}]$. The pendubot parameters are as follows.

The test on the pendubot was carried out as follows:

Links Inertias: $I_{1}=0.00053\left[\mathrm{Kg}-\mathrm{m}^{2}\right]$ and $I_{2}=0.00077$ $\left[\mathrm{Kg}-\mathrm{m}^{2}\right]$.

Mass of the links: $m_{1}=0.210[\mathrm{Kg}]$ and $m_{2}=0.1[\mathrm{Kg}]$. Length of the links: $l_{1}=0.15[\mathrm{~m}]$ and $l_{2}=0.3[\mathrm{~m}]$.

Distance to the center of mass: $l_{c_{1}}=0.12[\mathrm{~m}]$ and $l_{c_{2}}=$ $0.15[\mathrm{~m}]$.

Armature resistance: $k_{\tau}=0.0724[\Omega]$.

Torque constant: $\tau_{m}=2.983[\Omega]$.

The initial conditions are set as $x_{1}(0)=\pi / 2[\mathrm{rad}]$ and $x_{3}(0)=0[\mathrm{rad}]$. The desired trajectory consists of a rest to rest smooth trajectory, described as follows:

$$
\begin{aligned}
& y_{f}^{*}(t)=\frac{\beta_{2}+\beta_{3}}{\beta_{2}} x_{\delta 1}^{*}(t)+x_{\delta 3}^{*}(t), \\
& y_{f}^{*}(t)=\frac{\beta_{2}+\beta_{3}}{\beta_{2}}\left(\theta_{1}^{*}(t)-\frac{\pi}{2}\right)+\theta_{2}^{*}(t) .
\end{aligned}
$$

At $t=0$, the trajectory is set at $y_{f}^{*}(0)=0$; it implies that $\theta_{1}^{*}(t)=\pi / 2$ and $\theta_{2}^{*}(t)=0$; when the time reaches $t=4.5$, the desired trajectory moves to $y_{f}^{*}(6)=\left(\left(\beta_{2}+\beta_{3}\right) /\right.$ $\left.\beta_{2}\right)(\pi / 6)-(\pi / 6)$ with $\theta_{1}^{*}(t)=(2 / 3) \pi$ and $\theta_{2}^{*}(t)=-(\pi / 6)$ on a lapse of 2.5 seconds. Then, when time is $t=9.5[\mathrm{~s}]$, it moves to $y_{f}^{*}(13.5)=-\left(\left(\beta_{2}+\beta_{3}\right) / \beta_{2}\right)(\pi / 6)+(\pi / 6)$ with $\theta_{1}^{*}(t)=(\pi / 3)$ and $\theta_{2}^{*}(t)=-(\pi / 6)$ in 4 seconds, and finally, when $t=17.5[\mathrm{~s}]$, it returns to the initial position and remains in this position until the test is finished. Figure 15 shows the desired rest to rest positions of the pendubot system. 


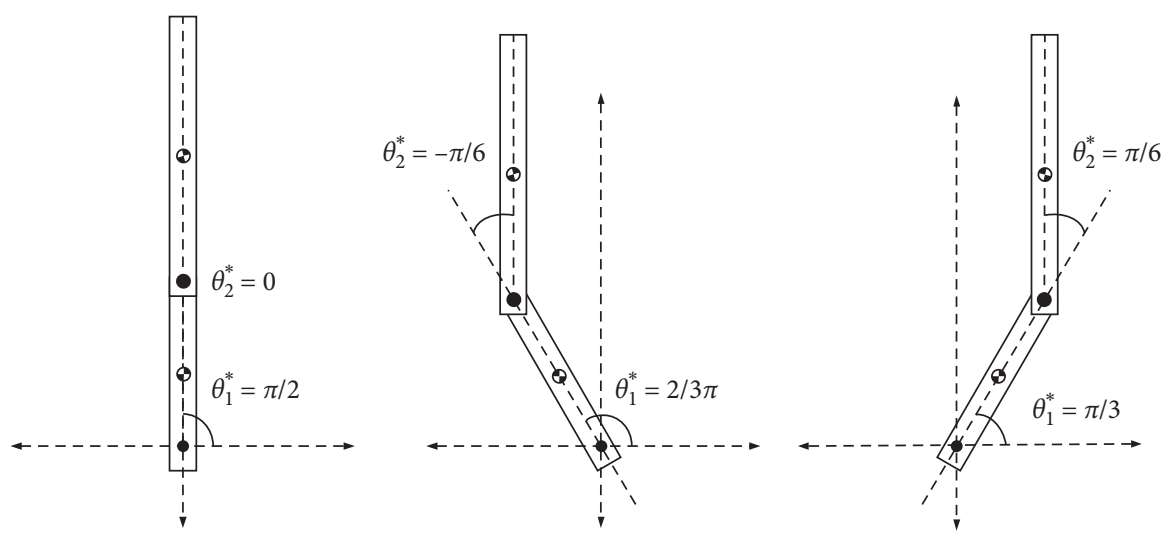

Figure 15: Pendubot desired rest to rest positions.

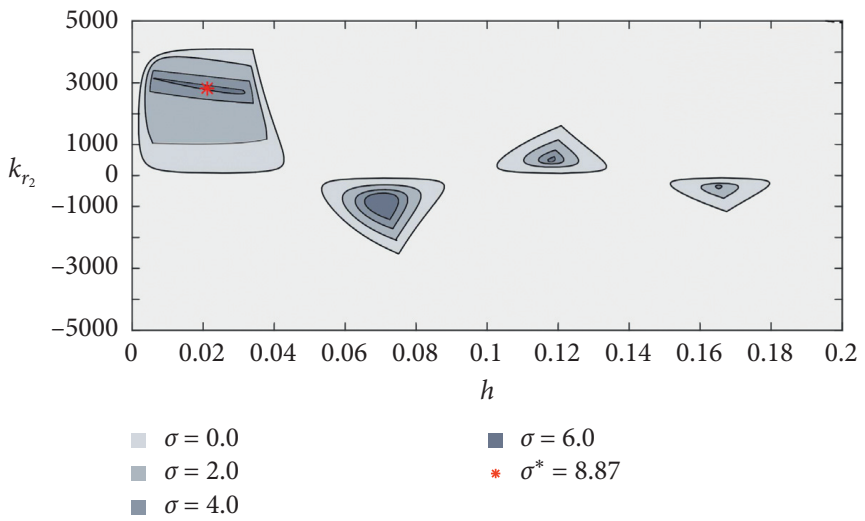

FIgURE 16: $\sigma$-stability boundaries.

The parameters of the controller were chosen according to (59 and 60), selecting $\omega_{c}=14$ and $\xi_{c}=1.2$ and then $k_{p_{1}}=460992, k_{p_{2}}=4562.88, k_{r_{1}}=422576$, and $\tau=0.0311$. The remainder of the parameters are calculated following Corollary 2, leading to $\sigma^{*}=8.8477, k_{r_{2}}=2832.39$, and $h^{*}=0.021$. The $\sigma$-stability boundaries are computed by means of Corollary 1, see Figure 16, where the maximal achievable decay rate $\sigma^{*}$ is marked as a red spot. Here, the zone outside the concentric regions corresponds to the unstable region. The set of gains for the feedback state controller were chosen as $\Phi=14$ and $\bar{\varsigma}=1.2$. Notice that the gain value coincides with the gain value used to select the proposed delayed control.

Figure 17 shows the flat output trajectory tracking performance of both controllers, the reference trajectory $y_{f}^{*}(t)$ is depicted in black line, the delayed controller response $y_{D}(t)$ is shown in the blue line, and the feedback controller response is shown in the red line $y_{\mathrm{FS}}(t)$, where it can be seen that the pendubot tracks the desired trajectory with adequate results, even when it is far from the equilibrium point. Figure 18 shows that the tracking errors $e_{D}(t)$ and $e_{\mathrm{FS}}(t)$ are bounded in an interval of approximately $[-0.025,0.025][\mathrm{rad}]$. Figures 19 and 20 show the variation of the positions of the links during the tracking trajectory task. Figure 21 exhibits the control input voltage $V_{D}$ calculated without using any time derivatives, which shows less noise

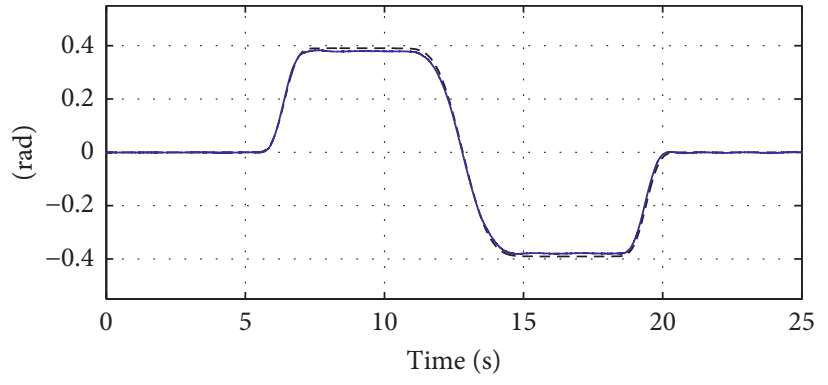

$---y_{f}^{*}(t)$

$-y_{f D}(t)$

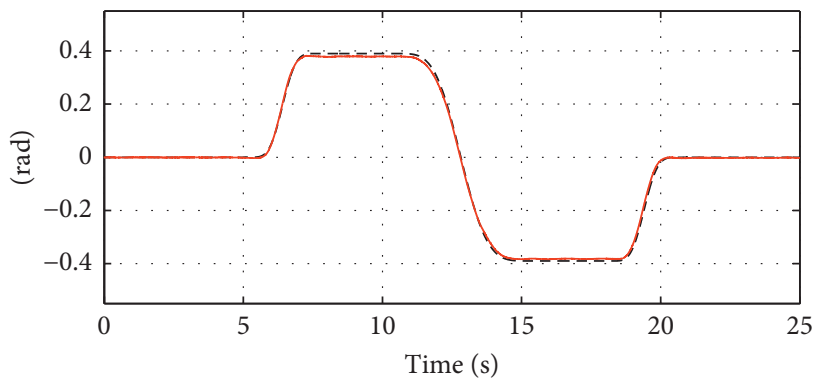

$---y_{f}^{*}(t)$

$-y_{f F S}(t)$

FIGURE 17: Flat output trajectory tracking. 

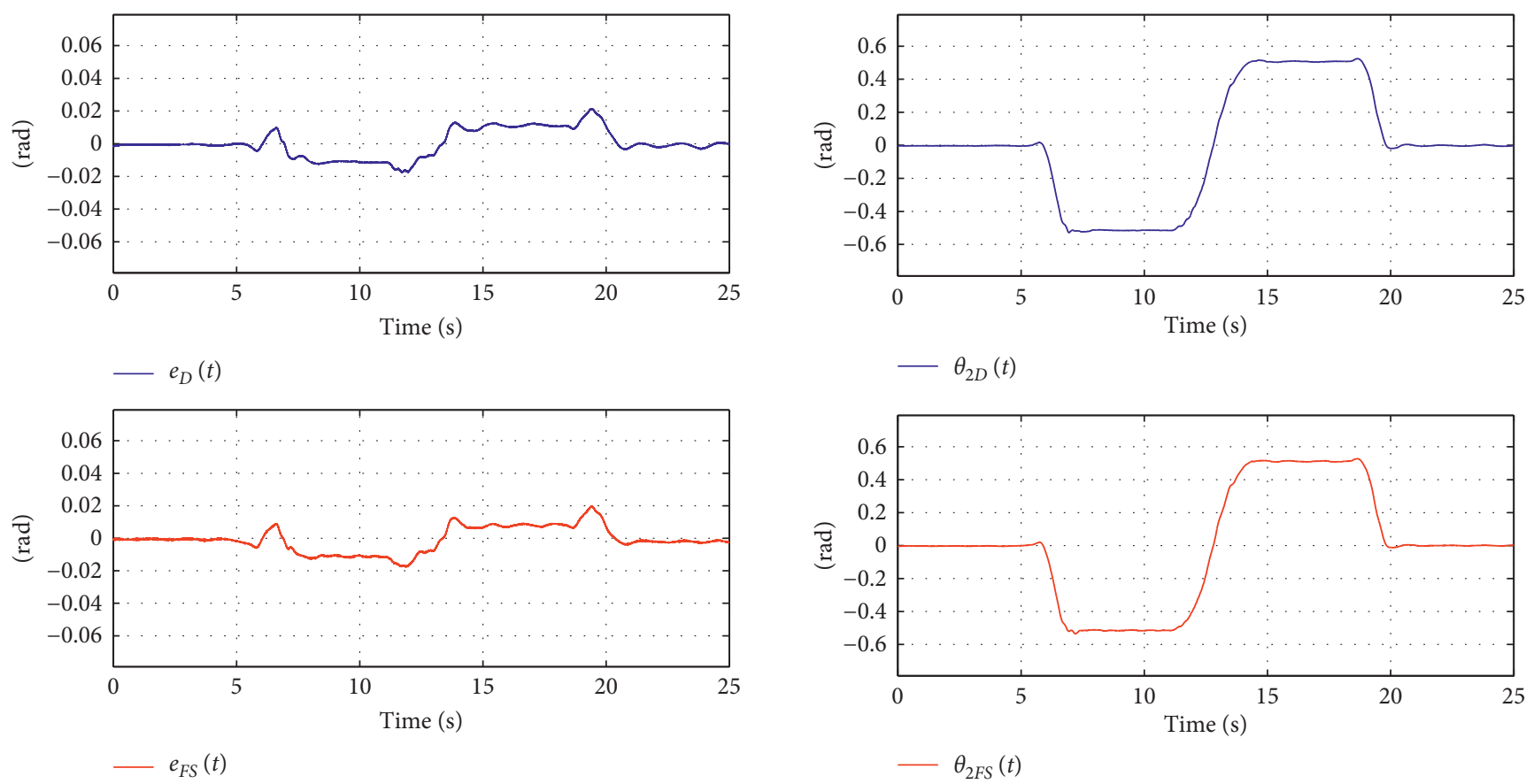

Figure 18: Tracking error.
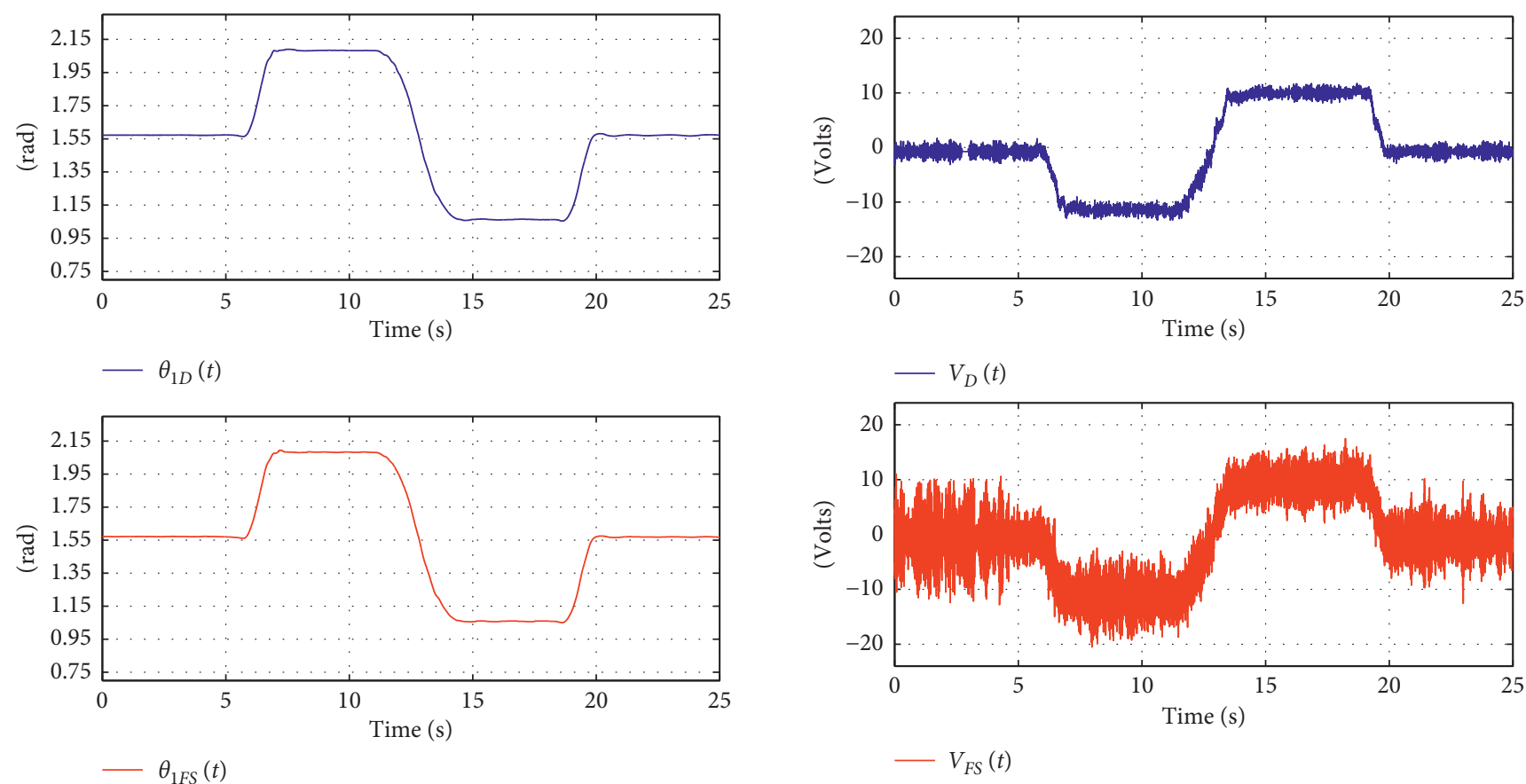

Figure 19: $\theta_{1}$ performance.

and less amplitude in contrast with $V_{\mathrm{FS}}$. The performance of both controllers is similar to good results; the main difference is on the voltage control. The Power Spectrum Density of $V_{D}$ and $V_{\mathrm{FS}}$ is depicted in Figure 22. Notice that $F S_{\text {PSD }}$ shows high-frequency components with a peak on 50

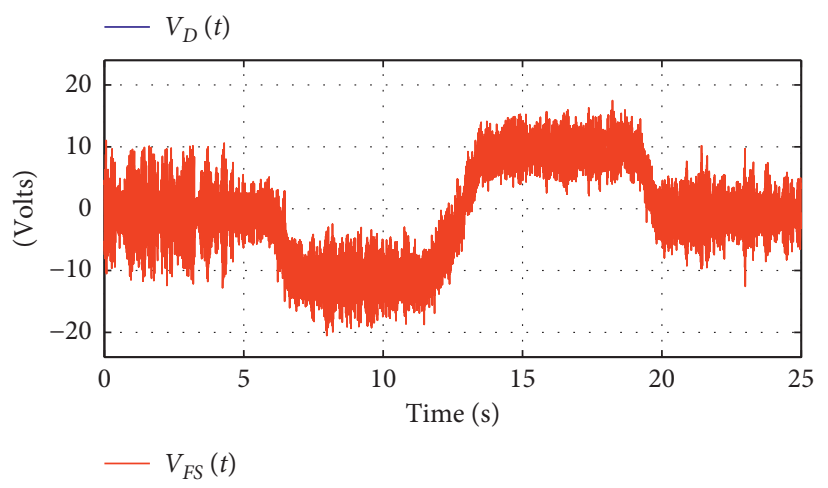

Figure 21: Control input voltage.

$[\mathrm{Hz}]$. Figure 23 shows an analysis using a quadratic index of the voltage, where $V_{\mathrm{FS}}$ consumes more energy. Thus, the proposed delay controller represents an attractive alternative to control a set of underactuated mechanical systems. A sensitivity analysis with a quadratic error index of the 

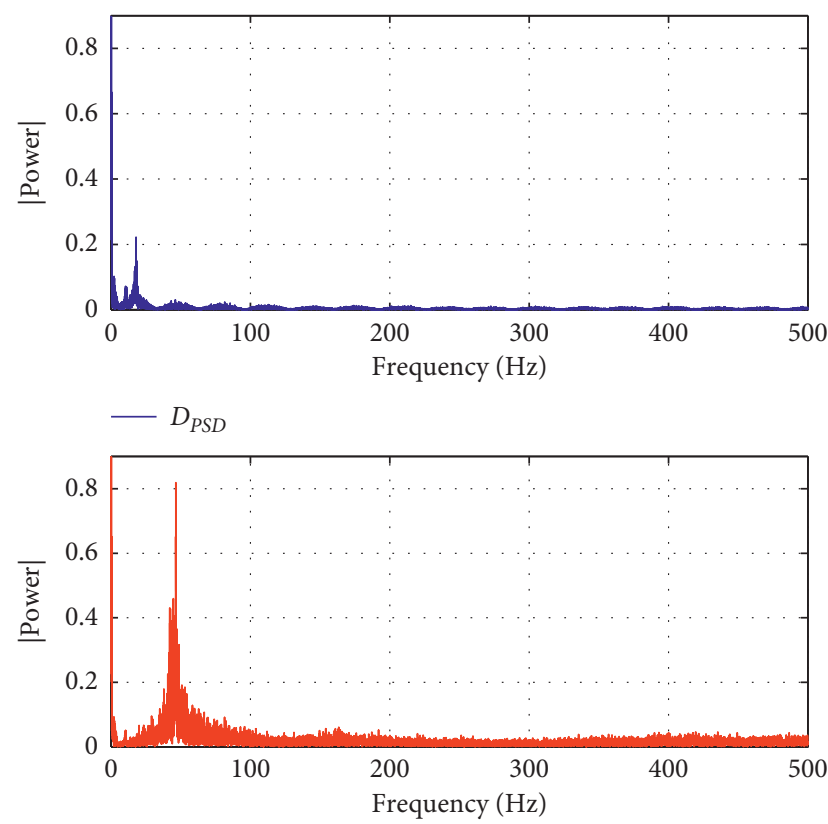

$-F S_{P S D}$

FIgURE 22: Power spectrum density.

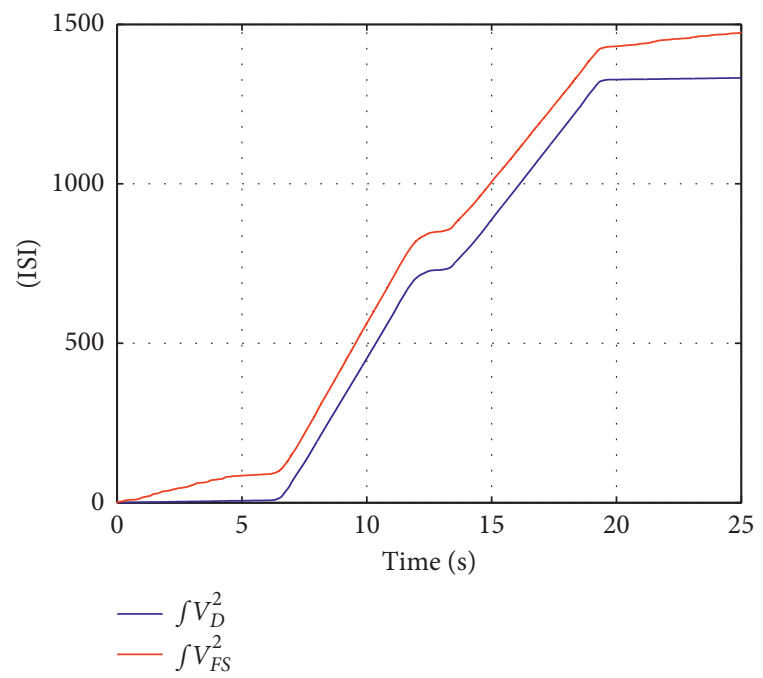

Figure 23: Performance index of the controller.

trajectory tasks was performed in simulation using a set of initial conditions for the angular positions $\theta_{1}(0)$ and $\theta_{2}(0)$. Figure 24 shows the first link performance; notice that the PR controller rank of initial conditions $\theta_{1 D}(0)$ remains on $[-8,8]$ degrees, in comparison with the FS controller rank of initial conditions $\theta_{1 \mathrm{FS}}(0)$ that remains on the interval $[-7,7]$ degrees. Similarly, Figure 25 shows the second link performance, the rank of the initial conditions for $\theta_{2 D}(0)$ is $[-12,12]$ degrees, while $\theta_{2 \mathrm{FS}}(0)$ is $[-11,11]$. Notice that the PR controller can control the pendubot system with further
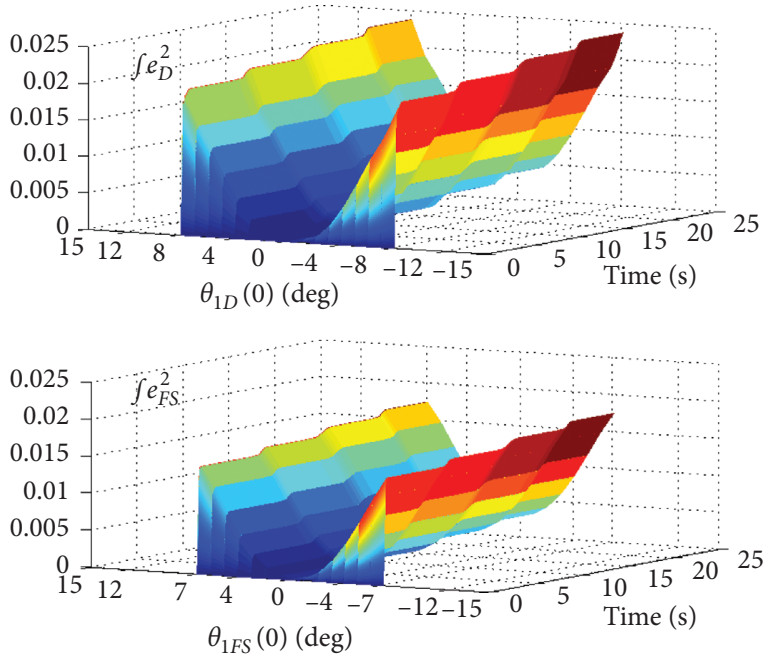

Figure 24: Assessment of the quadratic error index for the first link.
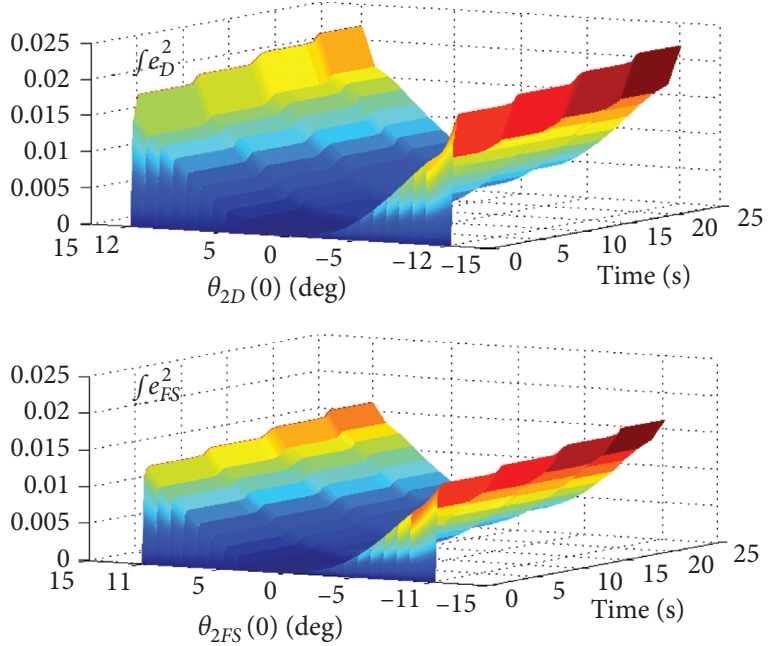

FIgURE 25: Assessment of the quadratic error index for the second link.

initial conditions from the equilibrium point than the PD controller.

\section{Conclusion}

In this paper, an alternative approach to control a class of underactuated systems of the fourth order through proportional retarded based controllers was proven and experimentally tested, leading to smooth tracking results thanks to the derivative-free control philosophy. The tuning procedure allows the practitioner to find appropriate control gains (the proposal is constructive). The cascade form of the tangent linearization of the underactuated system is an important design tool for the model simplification and the 
local controllability property for the design of a wide variety of controllers. Future research concerning the topic deals with the extension of the procedure to larger order systems as well as a more comprehensive development of the conditions involving all the control parameters. The case of study can be also extended for disturbed dynamics, in which integral actions can be proposed and tested.

\section{Data Availability}

All the data generated or analyzed during this study are included in this paper.

\section{Conflicts of Interest}

The authors declare that there are no conflicts of interest regarding the publication of this paper.

\section{Acknowledgments}

This paper is in memory of Oscar Villafuerte-Segura. This work was partially supported by "Secretaría de Investigación y Posgrado-IPN" under Grants SIP20201675 and SIP20201830, and CONACYT-México and InIAT Universidad Iberoamericana Ciudad de México.

\section{References}

[1] C. A. Ibañez and O. G. Frias, "Controlling the inverted pendulum by means of a nested saturation function," Nonlinear Dynamics, vol. 53, no. 4, pp. 273-280, 2008.

[2] C. A. Ibañez, O. G. Frias, and M. S. Castañón, "Lyapunovbased controller for the inverted pendulum cart system," Nonlinear Dynamics, vol. 40, no. 4, pp. 367-374, 2005.

[3] I. Fantoni, R. Lozano, and M. W. Spong, "Energy based control of the pendubot," IEEE Transactions on Automatic Control, vol. 45, no. 4, pp. 725-729, 2000.

[4] B. Lu, Y. Fang, and N. Sun, "Continuous sliding mode control strategy for a class of nonlinear underactuated systems," IEEE Transactions on Automatic Control, vol. 50, 2018.

[5] R. Xu and Ü. Özgüner, "Sliding mode control of a class of underactuated systems," Automatica, vol. 44, no. 1, pp. 233-241, 2008.

[6] R. Ortega, A. Loría, P. Nicklasson, and H. Sira-Ramirez, Passivity-based Control of Euler-Lagrange Systems: Mechanical, Electrical and Electromechanical Applications, Springer Science \& Business Media, Berlin, Germany, 1998.

[7] M. Spong, Underactuated Mechanical Systems, Springer, Berlin, Germany, 1998.

[8] J. Li, X. Qi, Y. Xia, and Z. Gao, “On asymptotic stability for nonlinear ADRC based control system with application to the ball-beam problem," 2016.

[9] M. Ramírez-Neria, H. Sira-Ramírez, R. Garrido-Moctezuma, and A. Luviano-Juárez, "Linear active disturbance rejection control of underactuated systems: the case of the furuta pendulum," ISA Transactions, vol. 53, no. 4, pp. 920-928, 2014.

[10] W. Tan and C. Fu, "Linear active disturbance-rejection control: analysis and tuning via imc," IEEE Transactions on Industrial Electronics, vol. 63, pp. 2350-2359, 2016.

[11] M. Reyhanoglu, A. van der Schaft, N. H. McClamroch, and I. Kolmanovsky, "Dynamics and control of a class of underactuated mechanical systems," IEEE Transactions on Automatic Control, vol. 44, no. 9, pp. 1663-1671, 1999.

[12] J. Hauser, S. Sastry, and P. Kokotovic, "Nonlinear control via approximate input-output linearization: the ball and beam example," IEEE Transactions on Automatic Control, vol. 37, no. 3, pp. 392-398, 1992.

[13] R. Olfati-Saber, "Normal forms for underactuated mechanical systems with symmetry," IEEE Transactions on Automatic Control, vol. 47, no. 2, pp. 305-308, 2002.

[14] M. Fliess, J. Lévine, P. Martin, and P. Rouchon, "Flatness and defect of non-linear systems: introductory theory and examples," International Journal of Control, vol. 61, no. 6, pp. 1327-1361, 1995.

[15] H. Sira-Ramírez, A. Luviano-Juárez, M. Ramírez-Neria, and E. W. Zurita-Bustamante, Active Disturbance Rejection Control of Dynamic Systems: A Flatness Based Approach, Butterworth-Heinemann, New York, NY, USA, 2017.

[16] N. S. Özbek and İ. Eker, "Investigation of time-delayed controller design strategies for an electromechanical system," IEEE, vol. 170, pp. 181-186, 2017.

[17] C. Abdallah, P. Dorato, J. Benites-Read, and R. Byrne, "Delayed positive feedback can stabilize oscillatory systems," 1993 American Control Conference, vol. 53, pp. 3106-3107, 1993.

[18] K. Gu, S.-I. Niculescu, and J. Chen, "On stability crossing curves for general systems with two delays," Journal of Mathematical Analysis and Applications, vol. 311, no. 1, pp. 231-253, 2005.

[19] J.-E. Hernández-Díez, C.-F. Méndez-Barrios, S. Mondié, S.-I. Niculescu, and E. J. González-Galván, "Proportionaldelayed controllers design for LTI-systems: a geometric approach," International Journal of Control, vol. 91, no. 4, pp. 907-925, 2018.

[20] C. Hwang and J. H. Hwang, "Stabilisation of first-order plus dead-time unstable processes using PID controllers," IEE Proceedings-Control Theory and Applications, vol. 151, no. 1, pp. 89-94, 2004.

[21] W. Just, T. Bernard, M. Ostheimer, E. Reibold, and H. Benner, "Mechanism of time-delayed feedback control," Physical Review Letters, vol. 78, no. 2, pp. 203-206, 1997.

[22] S.-I. Niculescu, K. Gu, and C. T. Abdallah, "Some remarks on the delay stabilizing effect in SISO systems," IEEE, vol. 3, 2003.

[23] N. Olgac, A. F. Ergenc, and R. Sipahi, “"Delay scheduling”: a new concept for stabilization in multiple delay systems," Journal of Vibration and Control, vol. 11, no. 9, pp. 1159-1172, 2005.

[24] K. Pyragas, "Continuous control of chaos by self-controlling feedback," Physics Letters A, vol. 170, no. 6, pp. 421-428, 1992.

[25] A. Ramírez, S. Mondié, R. Garrido, and R. Sipahi, "Design of proportional-integral-retarded (PIR) controllers for secondorder LTI systems," IEEE Transactions on Automatic Control, vol. 61 , no. 6, pp. 1688-1693, 2016.

[26] M. de Sousa Vieira and A. J. Lichtenberg, "Controlling chaos using nonlinear feedback with delay," Physical Review E, vol. 54, no. 2, pp. 1200-1207, 1996.

[27] G. M. Swisher and S. Tenqchen, "Design of proportionalminus-delay action feedback controllers for second-and thirdorder systems," IEEE, vol. 63, 1988.

[28] Q.-C. Zhong and H.-X. Li, "A delay-type PID controller," IFAC Proceedings Volumes, vol. 35, no. 1, pp. 265-270, 2002.

[29] S. Mondié, R. Villafuerte, and R. Garrido-Moctezuma, "Tuning and noise attenuation of a second order system using proportional retarded control," 2011.

[30] R. Villafuerte, S. Mondié, and R. Garrido, "Tuning of proportional retarded controllers: theory and experiments," IEEE 
Transactions on Control Systems Technology, vol. 21, no. 3, pp. 983-990, 2013.

[31] R. Villafuerte, S. Mondié, C. Vazquez, and J. Collado, "Proportional retarded control of a second order system," in Proceedings of the International Conference on Electrical Engineering, Computing Science and Automatic Control, CCE, New York, NY, USA, 2009.

[32] V. L. Kharitonov, S.-I. Niculescu, J. Moreno, and W. Michiels, "Static output feedback stabilization: necessary conditions for multiple delay controllers," IEEE Transactions on Automatic Control, vol. 50, pp. 82-86, 2005.

[33] F. Mazenc, S. Mondié, and S.-I. Niculescu, "Global stabilization of oscillators with bounded delayed input," Systems \& Control Letters, vol. 53, no. 5, pp. 415-422, 2004.

[34] S.-I. Niculescu and W. Michiels, "Stabilizing a chain of integrators using multiple delays," IEEE Transactions on Automatic Control, vol. 49, no. 5, pp. 802-807, 2004.

[35] D. A. Irofti, K. Gu, I. Boussaada, and S.-I. Niculescu, "Some insights into the migration of double imaginary roots under small deviation of two parameters," Automatica, vol. 88, pp. 91-97, 2018.

[36] M. W. Spong, P. Corke, and R. Lozano, "Nonlinear control of the reaction wheel pendulum," Automatica, vol. 37, no. 11, pp. 1845-1851, 2001.

[37] E. N. Gryazina, "The D-decomposition theory," Automation and Remote Control, vol. 65, no. 12, pp. 1872-1884, 2004.

[38] E. N. Gryazina and B. T. Polyak, "Stability regions in the parameter space: D-decomposition revisited," Automatica, vol. 42, no. 1, pp. 13-26, 2006.

[39] E. N. Gryazina, B. T. Polyak, and A. A. Tremba, "D-decomposition technique state-of-the-art," Automation and Remote Control, vol. 69, no. 12, pp. 1991-2026, 2008.

[40] Y. I. Neimark, "D-partition and robust stability," Computational Mathematics and Modeling, vol. 9, no. 2, pp. 160-166, 1998.

[41] J. Levine, Analysis and Control of Nonlinear Systems: A Flatness-Based Approach, Springer Science \& Business Media, Berlin, Germany, 2009.

[42] H. Sira-Ramirez and S. Agrawal, Differentially Flat Systems, CRC Press, New York, NY, USA, 2004.

[43] V. Kharitonov, Time-delay Systems: Lyapunov Functionals and Matrices, Springer Science \& Business Media, Berlin, Germany, 2012.

[44] J. I. Neimark, "D-decomposition of the space of quasipolynomials (on the stability of linearized distributive systems)," American Mathematical Society Translations, vol. 102, pp. 95-131, 1973.

[45] G. Escobar, R. Ortega, and H. Sira-Ramírez, "Output-feedback global stabilization of a nonlinear benchmark system using a saturated passivity-based controller," IEEE Transactions on Control Systems Technology, vol. 7, no. 2, pp. 289-293, 1999. 